\title{
COMPLEX VARIABLE TAUBERIANS
}

\author{
BY \\ SHMUEL AGMON
}

1. Introduction. The following is a known complex variable Tauberian due to Ikehara [3], used to give one of the simplest proofs to the prime number theorem:

THEOREM. Let $f(s)$ be analytic in the half-plane $\operatorname{Re}\{s\}>1$ and given there by:

$$
f(s)=\int_{0}^{\infty} e^{-s u} \phi(u) d u,
$$

where $\phi(u)$ is non-negative and nondecreasing. Suppose that

$$
\lim _{\sigma=+1}\left[f(\sigma+i t)-\frac{A}{\sigma-1+i t}\right]=\psi(t),
$$

uniformly in any interval $|t| \leqq T$, then

$$
\phi(u) \sim A e^{u} \quad(u \rightarrow \infty) .
$$

It is not difficult to show that Ikehara's theorem is equivalent to the following result:

THEOREM 1.1. Let $f(s)$ be represented in the half-plane $\operatorname{Re}\{s\}>0$ by:

$$
f(s)=\int_{0}^{\infty} e^{-s u} d \alpha(u),
$$

where $\alpha(u)$ is real and nondecreasing. Suppose that

$$
\lim _{\sigma=+0}\left[f(\sigma+i t)-\frac{A}{\sigma+i t}\right]=\psi(t)
$$

uniformly in any interval $|t| \leqq T$, then

$$
\alpha(u+h)-\alpha(u) \sim A h
$$$$
(u \rightarrow \infty) \text {, }
$$

uniformly for $0<h_{1} \leqq h \leqq h_{2}$ ( $h_{1}$ and $h_{2}$ being arbitrary positive constants).

Comparing the last theorem with the real variable Tauberian (HardyLittlewood), where one deduces from

(i) $f(s)$ is represented by (1.4) for $\operatorname{Re}\{s\}>0$, and

(ii) $f(\sigma) \sim A / \sigma$ for $\sigma \rightarrow+0$,

Presented to the Society, December 27, 1951; received by the editors April 7, 1952. 
that $\alpha(u) \sim A u$, one sees that the complex variable Tauberian gives the asymptotic growth of the "coefficient" $\alpha(u+h)-\alpha(u)$ while the real variable Tauberian gives only the asymptotic growth of the "partial sum" $\alpha(u)$. That one would obtain a better result in the complex case is of course to be expected in view of the much stronger assumptions underlaid in this case.

We propose to give in this paper, among other things, a generalization of Ikehara's theorem (stated in the form of Theorem 1.1). The most notable feature of our contribution will consist in dropping the condition that $s=0$ is essentially a simple pole. A rather general class of singularities will be admitted instead. The condition of monotonicity of $\alpha(u)$ will also be replaced by a more general condition. As an example of the type of results we shall obtain we cite the following theorem which is a special case of a more general result:

TheOREm 1.2. Let $f(s)$ be represented in the half-plane $\operatorname{Re}\{s\}>0$ by (1.4) where $\alpha(u)$ is nondecreasing. Let $f(s)$ be bounded in the strips $\sigma>0, \epsilon \leqq|t| \leqq T$, for any $0<\epsilon<T$. Suppose, furthermore, that there exists a function $\phi(s)$ analytic in $\operatorname{Re}\{s\}>0$ and given there by the Taylor-D series $\phi(s)=\sum a_{n} e^{-n s}$ such that:

(i) The only singularities of $\phi(s)$ on the imaginary axis are the points $2 \pi k i$ for $k=0, \pm 1, \cdots$.

(ii) $\lim \inf \left|a_{n}\right|>0$.

(iii) $\log \left|a_{n}\right|$ is a concave function of $n$.

(iv) For some $T_{0}>0, f(s)-\phi(s)$ is bounded in the half strip: $\sigma>0,|t| \leqq T_{0}$. Then

$$
\alpha(u+h)-\alpha(u) \sim h a_{[u]} \quad(u \rightarrow \infty),
$$

uniformly for $0<h_{1} \leqq h \leqq h_{2}$. (As usual we shall denote by $[u]$ the integral part of $u$ ).

Finally we mention that the method to be used in this paper differs from those used in similar problems and is more complex variable in character. It can be described as the method of principal indices and was introduced in [1] in problems concerning singularities of Dirichlet series. The following two sections will develop the basic results associated with this method.

2. The fundamental theorem. In this section we shall extend to Laplace transforms the Fundamental Theorem of [1]. We shall prove an important special case of the extension and shall then remark briefly on the proof of the general theorem. We shall establish

TheоREм 2.1. Let $f(s)$ be represented in the half-plane $\operatorname{Re}\{s\}>0$ by (1.4) and let $\sigma=0$ be the abscissa of absolute convergence of the Laplace transform. Put

$$
c_{n}=\int_{n}^{n+1}|d \alpha(u)|
$$


(Hence, $\lim \sup c_{n}^{1 / n}=1$.) Let $\left\{q_{n}\right\}$ be a sequence of positive numbers satisfying the following conditions:

(i) $q_{n} \geqq c_{n}$.

(2.2) (ii) $\lim \left(\log q_{n}\right) / n=0$.

(iii) $\log q_{n}$ is a concave function of $n: \log q_{n+1}-2 \log q_{n}+\log q_{n-1} \leqq 0$.

Put

$$
f_{x}(s)=\frac{f(s)-\int_{0}^{x} e^{-s u} d \alpha(u)}{q_{[x]} e^{-s x}} .
$$

Then, the family $\left\{f_{x}(s)\right\}$ is bounded uniformly in any compact region where $f(s)$ is analytic.

Proof. From (2.2) it follows that $q_{n} \uparrow$ and $q_{n+1} / q_{n} \downarrow 1$. Let

$$
\eta_{n}=\log q_{n+1}-\log q_{n} .
$$

Then, $\eta_{n} \geqq 0$ and $\eta_{n} \downarrow 0$. Hence, if $m$ and $n$ are two non-negative integers,

$$
\log q_{n+m}-\log q_{n} \leqq m \eta_{n}
$$

and

$$
\log q_{n+m}-\log q_{n} \geqq m \eta_{n+m-1} \geqq m \eta_{n+m} .
$$

Set

$$
g_{x}(s)=f_{x}\left(s+\eta_{[x]}\right) .
$$

Now, since $\eta_{n} \rightarrow 0$, any compact domain of regularity $D$ of $f(s)$ is also a domain of regularity for $g_{x}(s)$ if $x$ is taken sufficiently large. Since there is no loss of generality in proving the theorem for $x$ sufficiently large, we find that the theorem is equivalent to

$$
g_{x}(s)=O(1) \quad(x \rightarrow \infty) \text { uniformly for } s \in D \text { and } x \geqq x_{0} .
$$

By the Heine-Borel-Lebesgue, (2.7) will follow once the boundedness "in the small" of $\left\{g_{x}(s)\right\}$ is established. That is, once it is shown that with any $s_{0} \in D$ one can associate a neighborhood containing $s_{0}$ in which (2.7) holds. Now this, we claim, will follow from

$$
\left|g_{x}(s)\right| \leqq \frac{C}{\left|1-e^{-\sigma}\right|} \quad(C \text { being a constant }),
$$

for $s=\sigma+i t \in D$ and $x$ sufficiently large. Indeed, suppose that (2.8) holds. Then, obviously, $\left\{g_{x}(s)\right\}$ is locally uniformly bounded in the neighborhood of every point $s_{0}=\sigma_{0}+i t_{0} \in D$ with $\sigma_{0} \neq 0$ (since $\left.1-e^{-\sigma_{0}} \neq 0\right)$. On the other hand 
if $\sigma_{0}=0$ the boundedness follows by using a well known artifice: let $\delta>0$ be small enough so that $g_{x}(s)$ is analytic in the square $S_{2 \delta}:|\sigma| \leqq 2 \delta,\left|t-t_{0}\right| \leqq 2 \delta$ for all $x$ sufficiently large, and put

$$
G_{x}(s)=g_{x}(s)\left[s-i\left(t_{0}+2 \delta\right)\right]\left[s-i\left(t_{0}-2 \delta\right)\right] .
$$

It follows easily from (2.8) and (2.9) that on the sides of $S_{28}$ :

$$
\left|G_{x}(s)\right| \leqq C_{1},
$$

$C_{1}$ being a constant. But, by the maximum principle (2.10) must hold in the interior of $S_{2 \delta}$ as well. Restricting ourselves to the interior square $S_{\delta}$ we get: $\left|g_{x}(s)\right| \leqq C_{1} / \delta^{2}\left(x \geqq x_{0}\right)$ which is the desired local boundedness.

The theorem is thus reduced to establishing (2.8). We shall distinguish here two cases:

(i) $s=\sigma+i t$ with $\sigma>0$. In this case we get from (2.3) and (2.6)

$$
g_{x}(s)=\frac{\int_{x}^{\infty} e^{-(s+\eta[x]) u} d \alpha(u)}{q_{[x]} e^{-(s+\eta[x]) x}}=\frac{1}{q_{[x]}} \int_{0}^{\infty} e^{-(s+\eta[x]) u} d \alpha(x+u) .
$$

Hence

$$
\begin{aligned}
\left|g_{x}(s)\right| \leqq & \frac{1}{q_{[x]}}\left(\int_{0}^{1}|d \alpha(x+u)|+e^{-(\sigma+\eta[x])} \int_{1}^{2}|d \alpha(x+u)|+\cdots\right. \\
& \left.\left.+e^{-k(\sigma+\eta[x]}\right) \int_{k}^{k+1}|d \alpha(x+u)|+\cdots\right)
\end{aligned}
$$

Now, by (2.1) and (2.2),

$$
\begin{aligned}
\int_{k}^{k+1}|d \alpha(x+u)| & \leqq \int_{[x]+k}^{[x]+k+2}|d \alpha(u)|=c_{[x]+k}+c_{[x]+k+1} \\
& \leqq q_{[x]+k}+q_{[x]+k+1} \leqq 2 q_{[x]+k+1} .
\end{aligned}
$$

Thus, (2.12) and (2.11) give

$$
\begin{aligned}
\left|g_{x}(s)\right| \leqq 2\left[\frac{q_{[x]+1}}{q_{[x]}}+\frac{q_{[x]+2} e^{-\eta[x]}}{q_{[x]}} e^{-\sigma}\right. & +\cdots \\
& \left.+\frac{q_{[x]+k+1} e^{-k \eta[x]}}{q_{[x]}} e^{-k \sigma}+\cdots\right] .
\end{aligned}
$$

Using (2.5) for $n=[x]$ and $m=k+1$, we get: $\log q_{[x]+k+1}-\log q_{[x]}-k \eta_{[x]}$ $\leqq \eta_{[x]} \leqq \eta_{0}$. This and (2.13) yield

$$
\left|g_{x}(s)\right| \leqq 2 e^{\eta_{0}}\left(1+e^{-\sigma}+e^{-2 \sigma}+\cdots\right)=2 e^{\eta_{0}} /\left(1-e^{-\sigma}\right),
$$

which establishes (2.8) in the case under consideration. 
(ii) $s=\sigma+i t \in D$ with $\sigma<0$. In this case we may write

(2.14) $g_{x}(s)=\frac{f\left(s+\eta_{[x]}\right)}{q_{[x]} e^{-(s+\eta[x]) x}}-\frac{\int_{0}^{x} e^{-(s+\eta[x]) u} d \alpha(u)}{q_{[x]} e^{-(s+\eta[x]) x}}=I_{x}(s)-J_{x}(s)$.

Let $\delta>0$ be small enough so that $f(s)$ is still analytic in the closed region $D_{\delta}$ composed of all circles $|s-z| \leqq \delta$ with center $z$ in $D$. Put $M=\max |f(s)|$ for $s \in D_{\delta}$, and let $x$ be sufficiently large so that $\eta_{[x]}<\delta$. We then have for $s \in D$ with $\sigma<0$ :

$$
\left|I_{x}(s)\right| \leqq \frac{M}{q_{[x]} e^{-\sigma x} e^{-\eta[x] x}}<M e^{\eta[x]} e^{\eta[x][x]-\log q_{[x]}} .
$$

But $\eta_{[x]} \leqq \eta_{0}$ and, using $\left(2.5^{\prime}\right)$ for $m=[x]$ and $n=0$, we find that $[x] \eta_{[x]}$ $-\log q_{[x]} \leqq-\log q_{0}$. Hence

$$
\left|I_{x}(s)\right|<M e^{\eta_{0}} / q_{0}=C_{2} .
$$

On the other hand we obtain for $J_{x}(s)$, using (2.14), (2.2), and (2.1),

$$
\begin{aligned}
\left|J_{x}(s)\right|= & \frac{1}{q_{[x]}}\left|\int_{0}^{x} e^{(s+\eta[x])(x-u)} d \alpha(u)\right| \leqq \frac{1}{q_{[x]}} \int_{0}^{x} e^{(\sigma+\eta[x])(x-u)}|d \alpha(u)| \\
\leqq & \frac{1}{q_{[x]}}\left(\sum_{k=0}^{[x]-1} e^{(x-k-1) \sigma} e^{(x-k) \eta[x]} \int_{k}^{k+1}|d \alpha(u)|\right. \\
& \left.+e^{\eta[x]} \int_{[x]}^{x}|d \alpha(u)|\right) \\
\leqq & e^{\eta[x]}\left(\sum_{k=0}^{[x]-1} \frac{q_{k} e^{([x]-k) \eta[x]}}{q_{[x]}} e^{(x-k-1) \sigma}+1\right) .
\end{aligned}
$$

From $\left(2.5^{\prime}\right)$ we get (taking $m=[x]-k$ and $n=k$ ) that $\log q_{k}-\log q_{[x]}$ $+([x]-k) \eta_{[x]} \leqq 0$. Combining this with (2.17) (using also $\left.\eta_{[x]} \leqq \eta_{0}\right)$ we finally obtain

$$
\begin{aligned}
\left|J_{x}(s)\right| & \leqq e^{\eta[x]}\left(\sum_{k=0}^{[x]-1} e^{(x-k-1) \sigma}+1\right) \leqq e^{\eta[x]}\left(\sum_{k=0}^{[x]-1} e^{([x]-k-1) \sigma}+1\right) \\
& <e^{\eta[x]}\left(2+e^{\sigma}+e^{2 \sigma}+\cdots\right)=e^{\eta[x]}+\frac{e^{\eta[x]}}{1-e^{\sigma}} \\
& <e^{\eta_{0}}+\frac{e^{\eta_{0}}}{1-e^{\sigma}}<\frac{C_{3}}{\left|1-e^{-\sigma}\right|} .
\end{aligned}
$$

Thus, we have established (2.8) also in the last case and the proof of the theorem is complete. 
Theorem 2.1 is a particular case of a more general result. We remark, however, that in many applications this theorem suffices and there is no need to use the more general theorem (e.g. this is true in connection with the proof of Theorem 1.2). The case when Theorem 2.1 needs an improvement is when $c_{n} \rightarrow 0$. Indeed, in this case one would expect to have a corresponding result with a regular sequence $\left\{q_{n}\right\}$ satisfying $q_{n} \rightarrow 0$, whereas the conditions of regularity (2.2) imply that $\left\{q_{n}\right\}$ is nondecreasing and therefore is bounded away from zero. This defect is removed in the general theorem:

Theorem 2.2 (Fundamental Theorem). Suppose that the same assumptions as in Theorem 2.1 hold except that the conditions of regularity (ii) and (iii) are replaced by the following more general condition of regularity:

(ii) $q_{n} / q_{n+1} \rightarrow 1$.

(iii) $\log q_{n+1}-2 \log q_{n}+\log q_{n-1} \leqq C / n$ for some constant $C$.

Then, the conclusion of Theorem 2.1 holds.

We shall omit here the proof of Theorem 2.2 which is more laborious than that of Theorem 2.1. The corresponding result for a class of Dirichlet series was established in $[1$, p. 277] and the proof of Theorem 2.2 follows exactly the same lines. We shall only indicate the main tools used in the process of proof. One uses:

(a) An analogous theorem to Theorem 2.1 for entire functions $F(s)$ represented by everywhere absolutely converging Laplace transforms

$$
F(s)=\int_{0}^{\infty} e^{-s u} d A(u)
$$

(the analogue of Theorem II, p. 268 in [1]).

(b) The Borel transformation for Laplace transforms which associates with the function $f(s)$ of the theorem an entire function $F(s)$ represented by the everywhere absolutely converging Laplace transform:

$$
F(s)=\int_{0}^{\infty} e^{-s u} d A(u),
$$

where

$$
A(u)=\int_{0}^{u} \frac{d \alpha(v)}{\Gamma(v+1)}
$$

$(\Gamma(u)$ being the Euler gamma function). The two functions are related by

$$
f(\sigma+i t)=\int_{-\infty}^{\infty} e^{-\sigma^{\sigma-u}} e^{\sigma-u} F(u+i t) d u \quad \quad(\sigma>0) .
$$


We remark, however, that even in the case of Dirichlet series we have added here a trivial improvement to the result of [1] where it was assumed that the constant $C$ of $(2.18)$ is less than one. But this restriction is really superfluous and can be removed by a change of variable $s=s^{\prime} l$ with a suitably chosen constant $l$.

3. On the limit functions of the family $\left\{f_{x}(s)\right\}$. It follows from the Fundamental Theorem that the family of functions $\left\{f_{x}(s)\right\}$ defined by (2.3) is normal in any region where $f(s)$ is analytic. We shall now investigate the limit functions of this family and show that they have a number of interesting and simplifying properties.

THEOREM 3.1. Let $f(s)$ satisfy the conditions of Theorem 2.2 and suppose that the imaginary axis is not a natural boundary for $f(s)$. Let $\phi(s)$ be any limit function of $\left\{f_{x}(s)\right\}$. Then $\phi(s)$ has the following properties:

(i) $\phi(s)$ is analytic and uniform in the domain composed of the half-plane $\operatorname{Re}\{s\}>0$, the half-plane $\operatorname{Re}\{s\}<0$, and the regular points of $f(s)$ on the imaginary axis.

(ii) $\phi(s)$ has the representations

$$
\phi(s)=\int_{0}^{\infty} e^{-s u} d \beta_{+}(u) \quad \text { for } \operatorname{Re}\{s\}>0
$$

and

$$
\phi(s)=-\int_{0}^{\infty} e^{s u} d \beta_{-}(u) \quad \text { for } \operatorname{Re}\{s\}<0,
$$

where $\beta_{+}(u)$ and $\beta_{-}(u)$ are of bounded variation in any finite interval, and are given by

$$
\begin{aligned}
& \beta_{+}(u)=\lim _{k=\infty}\left(\frac{\alpha\left(x_{k}+u\right)-\alpha\left(x_{k}\right)}{q_{\left[x_{k}\right]}}\right) \\
& \beta_{-}(u)=\lim _{k=\infty}\left(\frac{\alpha\left(x_{k}\right)-\alpha\left(x_{k}-u\right)}{q_{\left[x_{k}\right]}}\right),
\end{aligned}
$$

$\left\{x_{k}\right\}$ being a sequence of increasing numbers tending to infinity. Furthermore, the variation of the functions $\beta_{ \pm}(u)$ in any interval $[a, b](0 \leqq a<b)$ verifies the inequality

$$
\int_{a}^{b}\left|d \beta_{ \pm}(u)\right| \leqq(b-a)+2 .
$$

(iii) If $s_{0}=i t_{0}$ is an isolated singularity of $f(s)$ on the imaginary axis (i.e. if $f(s)$ is regular for $\left.\sigma=0,\left|t-t_{0}\right| \leqq \delta, t \neq t_{0}\right)$, and if $s_{0}$ is also a singularity of $\phi(s)$, then it is a simple pole for $\phi(s)$. 
Proof. Let us pick a simply connected domain $D$ such that: (a) it is a domain of analyticity of $f(s)$, and (b) it contains all the regular points of $f(s)$ on the imaginary axis. Since $\left\{f_{x}(s)\right\}$ is normal in $D$ it will follow that any limit function $\phi(s)$ is analytic in $D$. We shall first establish property (ii) for the points of $D$. We shall denote throughout this paper by $V_{a}^{b}(\alpha)$ the total variation of $\alpha(u)$ in $[a, b]$ :

$$
V_{a}^{b}(\alpha)=\int_{a}^{b}|d \alpha(u)|
$$

Let, now, $s$ be any point in the half-plane $\operatorname{Re}\{s\}>0$. Using (2.3) and (1.4) we can write:

$$
f_{x}(s)=\frac{1}{q_{[x]} e^{-x s}} \int_{x}^{\infty} e^{-s u} d \alpha(u)=\int_{0}^{\infty} e^{-s u} d \alpha_{x}(u)
$$

where

$$
\alpha_{x}(u)=\frac{\alpha(u+x)-\alpha(x)}{q_{[x]}} .
$$

We thus find for $0 \leqq a<b$, using (3.6), (3.4), (2.1), and (2.2) (i), that

$$
\begin{aligned}
V_{a}^{b}\left(\alpha_{x}\right) & =\frac{1}{q_{[x]}} V_{a+x}^{b+x}(\alpha) \leqq \frac{1}{q_{[x]}} V_{[a+x]}^{[b+x]+1}(\alpha) \\
& \leqq \frac{1}{q_{[x]}}\left(q_{[a+x]}+q_{[a+x]+1}+\cdots+q_{[b+x]}\right) .
\end{aligned}
$$

From $q_{n+1} / q_{n} \rightarrow 1$ and (3.7) we get

(3.8) $\lim \sup V_{a}^{b}\left(\alpha_{x}\right) \leqq \lim \sup ([b+x]-[a+x]+1) \leqq(b-a)+2$.

Let us define the function $p(x)$ by

$$
\log p(x)=\max _{0 \leqq \lambda \leqq x} \max _{\mu \geqq 0}\left|\log q_{\lambda+\mu}-\log q_{\mu}\right| .
$$

It is readily seen that $p(x)$ is nondecreasing $(p(x) \geqq 1)$. Furthermore, from $q_{n+1} / q_{n} \rightarrow 1$ it follows that $\lim [(\log p(x)) / x]=0$. Hence, taking in (3.7) $b=a+1$ and using (3.9) we find that

$$
V_{a}^{a+1}\left(\alpha_{x}\right) \leqq \frac{q_{[a+x]}}{q_{[x]}}+\frac{q_{[a+x]+1}}{q_{[x]}} \leqq p(a+1)+p(a+2) \leqq 2 p(a+2) .
$$

From (3.10) it is seen that the family $\left\{\alpha_{x}(u)\right\}$ is uniformly of bounded variation in any finite interval. Furthermore, we have a uniform estimate for the variation in $[a, a+1]$ in terms of the function $p(x)$. 
Suppose, now, that the limit function $\phi(s)$ is given by

$$
\phi(s)=\lim _{k=\infty} f_{x_{k}}(s) .
$$

Apply the above results to the sequence $\left\{\alpha_{x_{k}}(u)\right\}$. By a well known theorem due to Helly it is possible to extract a subsequence $\left\{\alpha_{x_{k^{\prime}}}(u)\right\}$ which converges to a function $\beta_{+}(u)$ on the positive axis, $\beta_{+}(u)$ being of bounded variation in any finite interval. To simplify the notation assume that $\left\{\alpha_{x_{k}}(u)\right\}$ already has this property, so that

$$
\beta_{+}(u)=\lim _{k=\infty} \alpha_{x_{k}}(u)=\lim _{k=\infty}\left(\frac{\alpha\left(u+x_{k}\right)-\alpha\left(x_{k}\right)}{q_{\left[x_{k}\right]}}\right) .
$$

From (3.8) (since $V_{a}^{b}\left(\beta_{+}\right) \leqq \lim \sup V_{a}^{b}\left(\alpha_{x_{k}}\right)$ ) we get $V_{a}^{b}\left(\beta_{+}\right) \leqq b-a+2$ which we note in connection with the inequality (3.3) for $\beta_{+}(u)$. Now, by a theorem due to Helly and Bray it follows that if $g(u)$ is any continuous function in $[a, b]$, then

$$
\lim \int_{a}^{b} g(u) d \alpha_{x_{k}}(u)=\int_{a}^{b} g(u) d \beta_{+}(u) .
$$

Taking $g(u)=e^{-s u}$, using also (3.10) and the properties of $p(x)$, we easily obtain that the last result is also valid for $b=\infty$, so that

$$
\phi(s)=\lim _{k=\infty} \int_{0}^{\infty} e^{-s u} d \alpha_{x_{k}}(u)=\int_{0}^{\infty} e^{-s u} d \beta_{+}(u) \text { for } \operatorname{Re}\{s\}>0 .
$$

This is nothing else but (3.1) and we see that (ii) is true for all points $s$ in the half-plane $\operatorname{Re}\{s\}>0$.

Consider now the case where $s=\sigma+i t \in D$ with $\sigma<0$. In this case we may write

$$
f_{x}(s)=\frac{f(s)}{q_{[x]} e^{-s x}}-\frac{1}{q_{[x]} e^{-s x}} \int_{0}^{x} e^{-s u} d \alpha(u) .
$$

But $f(s) /\left(q_{[x]} e^{-s x}\right) \rightarrow 0$ (since $\sigma<0$ and $q_{n}^{1 / n} \rightarrow 1$ ). We therefore have

$$
\phi(s)=-\lim _{k=\infty}\left(\frac{1}{q_{\left[x_{k}\right]} e^{-s x_{k}}} \int_{0}^{x_{k}} e^{-s u} d \alpha(u)\right)=-\lim _{k=\infty} \int_{0}^{x_{k}} e^{s u} d \bar{\alpha}_{x_{k}}(u),
$$

where

$$
\bar{\alpha}_{x}(u)=\frac{\alpha(x)-\alpha(x-u)}{q_{[x]}} \quad(0 \leqq u \leqq x) .
$$

Now it is easily seen that the relations (3.8) and (3.10) are also satisfied by $\left\{\bar{\alpha}_{x}(u)\right\}$. (One should, however, continue the definition of $\bar{\alpha}_{x}(u)$ outside the 
interval $[0, x]$ by putting $\bar{\alpha}_{x}(u)=0$ for $u>x$.) We could therefore again extract a subsequence from $\left\{\bar{\alpha}_{x_{k}}(u)\right\}$ which will converge on the positive axis to a function $\beta_{-}(u)$ of bounded variation in any finite segment. We may assume that $\left\{\bar{\alpha}_{x_{k}}(u)\right\}$ itself has the convergence property and that

$$
\beta_{-}(u)=\lim _{k=\infty} \bar{\alpha}_{x_{k}}(u)=\lim _{k=\infty}\left(\frac{\alpha\left(x_{k}\right)-\alpha\left(x_{k}-u\right)}{q_{\left[x_{k}\right]}}\right) .
$$

We shall also find from (3.15), (3.13), and (3.10), that

$$
\phi(s)=-\lim _{k=\infty} \int_{0}^{x_{k}} e^{s u} d \bar{\alpha}_{x_{k}}(u)=-\int_{0}^{\infty} e^{s u} d \beta_{-}(u),
$$

which is $\left(3.1^{\prime}\right)$. From (3.15) and (3.8) we also see that we get the corresponding relations (3.2) and (3.3) for $\beta_{-}(u)$. This establishes (ii) for $s \in D$. However, on account of (3.3) the integral $\left(3.1^{\prime}\right)$ converges absolutely for $\operatorname{Re}\{s\}$ $<0$. It gives the analytic continuation of $\phi(s)$ in this half-plane. Hence the truth of (i) is also established. Finally, to establish property (iii) we have only to note that from property (i) it follows that $\phi(s)$ is single-valued around $s=s_{0}$ so that the singularity can be only a pole or an essential singularity. $\phi(s)$ must also satisfy (using (3.3)) an inequality of the type

$$
|\phi(s)| \leqq A+\frac{B}{|\sigma|},
$$

where $A$ and $B$ are constants. It is now a standard technique (see [1, p. 284]) to show that these properties imply that $s_{\theta}$ must be a simple pole. This completes the proof of the theorem.

We next take the important question which remained unsettled in the last theorem, namely: under what conditions may one assert that not all the limit functions $\phi(s)$ are identically zero, or even do not reduce to a constant. For, one would hardly expect that Theorem 3.1 will be of any use if the limit process will always yield a function which is identically zero. It is at this stage that one has to restrict the class of functions $\alpha(u)$. In this paper we shall consider two classes which we shall denote by $\Delta$ and $\Omega$ defined in the following manner.

Definition 3.1. A function $\alpha(u)(u \geqq 0)$ will be said to belong to the class $\Delta$ if there exist a positive number $d$ and a positive number $\gamma$ less than $\pi$ such that: for any fxed positive number $u_{0}$ and any $u_{1}$ and $u_{2}$ in the interval $\left[u_{0}-d, u_{0}+d\right]$ such that $u_{1}<u_{2}$, the points $\alpha\left(u_{2}\right)-\alpha\left(u_{1}\right)$ will all lie in an angle $\Sigma_{u_{0}}$ of the complex plane having its vertex at the origin and whose opening is less than $\gamma$.

Definition 3.2. A function $\alpha(u)(u \geqq 0)$ will be said to belong to the class $\Omega$ if given $\epsilon>0$ (small) and $N>0$ (large), then there exists a number $u_{0}=u_{0}(\epsilon, N)$ such that all the points $\alpha(u+x)-\alpha(u)$ for $u \geqq u_{0}$ and $0<x \leqq N$ will lie in an angle of the complex plane (depending on $u$ and $N$ but not on $x$ ) having its 
vertex at the origin and whose opening is less than $\epsilon$.

It is readily seen that $\Omega$ is a subclass of $\Delta$. (Nevertheless, $\Omega$ contains as a subclass the class of monotonic functions which is the class generally considered in Tauberian theorems.) We shall also introduce the notion of a sequence of principal indices:

DeFINITION 3.3. Let $f(s)$ be represented in the half-plane $\operatorname{Re}\{s\}>0$ by (1.4) ( $\sigma=0$ being the abscissa of absolute convergence). Let $\left\{c_{n}\right\}$ be defined by (2.1). Then a sequence $\left\{x_{k}\right\}, 0 \leqq x_{k} \uparrow \infty$, will be said to be a sequence of principal indices if the following holds: there exists a regular sequence $\left\{q_{n}\right\}$ satisfying (2.18) such that

(i) $q_{n} \geqq c_{n}$.

(ii) $\delta q_{n_{k}} \leqq c_{n_{k}}$ for some subsequence $\left\{n_{k}\right\}, k=1,2, \cdots$, and $\delta>0$.

(iii) $x_{k}=n_{k}+O(1)(k \rightarrow \infty)$.

Now in $[1$, p. 274] it was shown that given any sequence of positive numbers $\left\{c_{n}\right\}$ with $\lim \sup c_{n}^{1 / n}=1$ one can find a sequence $\left\{q_{n}\right\}$ satisfying: (i) $q_{n} / q_{n+1} \rightarrow 1$. (ii) $\lim \sup n\left(\log q_{n+1}-2 \log q_{n}+\log q_{n-1}\right) \leqq 0$. (iii) $q_{n} \geqq c_{n}$. (iv) $q_{n_{k}}=c_{n_{k}}$ for an infinite subsequence. Hence $\left\{q_{n}\right\}$ of the lemma is a "regular majorant" and the corresponding sequence $\left\{n_{k}\right\}$ is a sequence of principal indices. Thus, we may assert that

Corollary. Any function $f(s)$ satisfying the conditions of Definition 3.3 has a sequence of principal indices.

We can now answer the question raised above and give conditions under which limit functions $\phi(s)$ of a suitably defined normal family $\left\{f_{x}(s)\right\}$ exist which do not reduce to a constant. As a matter of fact we shall establish more and prove

THEOREM 3.2. Let $f(s)$ satisfy the conditions of Theorem 3.1 and suppose, furthermore, that

(a) $\alpha(u)$ belongs to the class $\Delta$ (see Definition 3.1).

(b) $\left\{x_{k}\right\}$ is a sequence of principal indices.

(c) The sequence $\left\{q_{n}\right\}$ is precisely that sequence associated with $\left\{x_{k}\right\}$ in Definition 3.3.

(d) $\left\{f_{x_{k}}(s)\right\}$ is the subsequence of (2.3) corresponding to the sequences (b) and $(c)$.

Then, any limit function $\phi(s)$ of the normal family $\left\{f_{x_{k}}(s)\right\}$ has at least one singular point on the imaginary axis. (Thus, in particular, $\phi(s)$ is not a constant.)

Proof. We shall first prove the theorem in the case where $\alpha(u)$ is real and nondecreasing, in which case the proof is particularly simple. We note, however, that the same proof almost word by word is also valid when $\alpha(u)$ belongs to the class $\Omega$. Making use of the hypotheses of the theorem and our additional assumption we may write: 


$$
0 \leqq \alpha(n+1)-\alpha(n) \leqq q_{n}
$$$$
(n=1,2, \cdots) \text {. }
$$

Now, since $\left\{x_{k}\right\}$ is a sequence of principal indices, there exists a sequence of integers $\left\{n_{k}\right\}$ with $x_{k}=n_{k}+O(1)$, and a constant $\delta>0$, such that

$$
\alpha\left(n_{k}+1\right)-\alpha\left(n_{k}\right) \geqq \delta q_{n_{k}} \quad(k=1,2, \cdots) .
$$

But $\left\{n_{k}\right\}$ itself is a sequence of principal indices and, we claim, it is enough to establish the theorem for this particular sequence. Indeed, we have the relation

$$
\begin{aligned}
f_{x_{k}}(s)= & \frac{f(s)-\int_{0}^{x_{k}} e^{-s u} d \alpha(u)}{q_{\left[x_{k}\right]} e^{-s x_{k}}}=\frac{\int_{x_{k}}^{n_{k}} e^{-s u} d \alpha(u)}{q_{\left[x_{k}\right]} e^{-x_{k} s}} \\
& +\frac{q_{n_{k}} e^{\left(x_{k}-n_{k}\right) s}}{q_{\left[x_{k}\right]}} \cdot \frac{f(s)-\int_{0}^{n_{k}} e^{-s u} d \alpha(u)}{q_{n_{k}} e^{-n_{k} s}}=I_{k}(s)+\frac{q_{n_{k}}}{q_{\left[x_{k}\right]}} e^{r_{k} s} f_{n_{k}}(s)
\end{aligned}
$$

where $I_{k}(s)$ is entire and uniformly bounded in any finite domain. Also $r_{k}=x_{k}-n_{k}=O(1)$, and $q_{n_{k}} / q_{\left[x_{k}\right]} \rightarrow 1$. It follows now readily from the above that with any limit function $\phi(s)$ of the family $\left\{f_{x_{k}}(s)\right\}$ one may associate a limit function $\phi^{*}(s)$ of the family $\left\{f_{n_{k}}(s)\right\}$ such that

$$
\phi(s)=I(s)+e^{r s} \phi^{*}(s),
$$

where $I(s)$ is entire and $r$ is a real number. This establishes the above contention since $\phi(s)$ and $\phi^{*}(s)$ will have the same singularities. Now if $\phi(s)$ is a limit function of $\left\{f_{n_{k}}(s)\right\}$, it possesses by Theorem 3.1 the two representations (3.1) and $\left(3.1^{\prime}\right)$ where the functions $\beta_{+}(u)$ and $\beta_{-}(u)$ are given by

$$
\begin{aligned}
& \beta_{+}(u)=\lim _{\boldsymbol{k}^{\prime}=\infty}\left(\frac{\alpha\left(n_{k^{\prime}}+u\right)-\alpha\left(n_{k^{\prime}}\right)}{q_{n_{k^{\prime}}}}\right), \\
& \beta_{-}(u)=\lim _{k^{\prime}=\infty}\left(\frac{\alpha\left(n_{k^{\prime}}\right)-\alpha\left(n_{k^{\prime}}-u\right)}{q_{n_{k^{\prime}}}}\right) .
\end{aligned}
$$

(We denote by $\left\{k^{\prime}\right\}$ a certain subsequence of the integers.) But, since $\alpha(u)$ is nondecreasing, it follows from (3.21) that the functions $\beta_{+}(u)$ and $\beta_{-}(u)$ are likewise nondecreasing. They also vanish at the origin. Furthermore, from (3.19) and (3.21) it follows that $\beta_{+}(u)$ is not identically zero since

$$
\beta_{+}(1) \geqq \liminf _{k=\infty}\left(\frac{\alpha\left(n_{k}+1\right)-\alpha\left(n_{k}\right)}{q_{n_{k}}}\right) \geqq \delta>0 .
$$

We shall now show that all the properties of $\phi(s)$ derived above imply that $\phi(s)$ is not a constant. Indeed, suppose by way of contradiction that $\phi(s)$ is a constant. Then, from the representations (3.1) and (3.1') (using the 
uniqueness theorem for Laplace transforms) it will follow that at all respective points of continuity $\beta_{+}(u)=c_{+}$and $\beta_{-}(u)=c_{-}$where $c_{+}$and $c_{-}$are non-negative constants. But, as we have noted above, the functions $\beta_{+}(u)$ and $\beta_{-}(u)$ are nondecreasing. One deduces now easily that one must have everywhere on the positive axis (since the points of continuity are everywhere dense):

$$
\beta_{+}(u) \equiv c_{+} \geqq 0 \quad \text { and } \quad \beta_{-}(u) \equiv c_{-} \geqq 0 .
$$

Combining this with (3.22) we also find that $c_{+}=\beta_{+}(1)>0$ (so that $c_{+}$is actually positive). But, now, if we return to the representations (3.1) and $\left(3.1^{\prime}\right)$ making use of (3.23) and $\beta_{ \pm}(0)=0$, we get:

$$
\phi(s)=c_{+}>0 \text { for } \operatorname{Re}\{s\}>0 \text { and } \phi(s)=-c_{-} \leqq 0 \text { for } \operatorname{Re}\{s\}<0 .
$$

This, clearly, leads to the contradiction $0<c_{+}=-c_{-} \leqq 0$, and establishes the nonconstancy of $\phi(s)$.

Finally, let us show that $\phi(s)$ must have at least one singular point on the imaginary axis. Indeed, otherwise $\phi(s)$ would be an entire function. Furthermore, it will satisfy an inequality of the type (3.17). But, it is easy to show that (3.17) implies the boundedness of $\phi(s)$ in the whole plane and this in turn implies that $\phi(s)$ is a constant. We are back at the case which we have proved to be impossible. This completes the proof of the theorem in the case where $\alpha(u)$ is nondecreasing.

We would like to point out that in the special case considered above one shows easily that the origin must always be a singularity of $\phi(s)$. Indeed, for $\sigma>0$,

$$
\phi(\sigma) \geqq \int_{0}^{1} e^{-\sigma u} d \beta_{+}(u)
$$

so that

$$
\limsup _{\sigma=+0} \phi(\sigma) \geqq \int_{0}^{1} d \beta_{+}(u)=\beta_{+}(1) \geqq \delta>0,
$$

while, on the other hand, for $\sigma<0$ :

$$
\limsup _{\sigma=-0} \phi(\sigma)=-\limsup _{\sigma=-0} \int_{0}^{\infty} e^{\sigma u} d \beta_{-}(u) \leqq 0 .
$$

This demonstrates that $\phi(s)$ cannot even be continuous at $s=0$. We further note that since any singular point of $\phi(s)$ must also be a singularity of $f(s)$ we have incidentally proved a theorem due to Landau. A less trivial result is obtained if we use our previous remark that the same method is effective in case $\alpha(u)$ belongs only to the class $\Omega$ (the limit functions in this last case except for a constant multiplier will have all the properties of the functions $\phi(s)$ discussed above). We shall then obtain the following generalization of 
Landau's theorem: if $\alpha(u)$ belongs to the class $\Omega$, then the real point on the axis of convergence of (1.4) is a singularity of $f(s)$.

We pass now to the proof of the general case. It will be assumed only that $\alpha(u)$ belongs to the class $\Delta$. Let $d$ be the positive constant associated with $\alpha(u)$ by Definition 3.1 and let $\eta$ be a number satisfying $0<\eta<d$. We shall first show that there exists a sequence of principal indices $\left\{x_{k}^{\prime}\right\}$ such that $x_{k}^{\prime}=x_{k}+O(1)$ and

$$
V_{x_{k^{\prime}}-\eta}^{x_{k^{\prime}}+\eta}(\alpha) \geqq c q_{\left[x_{k^{\prime}}\right]}
$$

for some positive constant $c$ and for all $k$ sufficiently large $\left(V_{a}^{b}(\alpha)\right.$ has the meaning of (3.4)). Indeed, from Definition 3.3 it follows that there exists a sequence of integers $\left\{n_{k}\right\}$ and a positive constant $\delta$ satisfying:

$$
x_{k}=n_{k}+O(1) \quad \text { and } \quad V_{n_{k}}^{n_{k}+1}(\alpha) \geqq \delta q_{n_{k}} .
$$

Choose a positive integer $p$ such that $1 / 2 p \leqq \eta$; divide the interval $\left[n_{k}, n_{k}+1\right]$ into $p$ equal parts, and denote by $x_{k}^{\prime}$ the center of this subinterval over which the variation of $\alpha(u)$ is the maximum. This sequence will have the desired properties. Indeed, $\left|x_{k}^{\prime}-n_{k}\right|<1$ and

$$
V_{x_{k^{\prime}-\eta}}^{x_{k^{\prime}+\eta}}(\alpha) \geqq V_{x_{k^{\prime}-1 / 2 p}}^{x_{k^{\prime}+1 / 2 p}}(\alpha) \geqq \frac{1}{p} V_{n_{k}}^{n_{k+1}}(\alpha)>c q_{\left[x_{k^{\prime}}\right]},
$$

which will give (3.24) if one chooses $0<c<\delta / p$ and $k$ sufficiently large. Now, by an argument similar to the one used in the proof of the special case (which gave the relation (3.20)) one can show that it is enough to prove the theorem for the family $\left\{f_{x_{k^{\prime}}}(s)\right\},\left\{x_{k}^{\prime}\right\}$ being the associated sequence of principal indices just introduced. To simplify the notations we shall assume that $\left\{x_{k}\right\}$ already has the desired properties. Let $\phi(s)$ be a limit function of $\left\{f_{x_{k}}(s)\right\}$. Once more we shall simplify the notation by assuming that

$$
\lim _{\boldsymbol{k}=\infty} f_{x_{k}}(s)=\phi(s) \text {. }
$$

Furthermore, choosing if necessary a subsequence of the $x_{k}$ 's, we may assume (because of (3.24)) that we always have either

$$
V_{x_{k}}^{x_{k}+\eta}(\alpha) \geqq \frac{c}{2} q_{\left[x_{k}\right]} \quad\left(k \geqq k_{0}\right)
$$

or

$$
V_{x_{k}-\eta}^{x_{k}}(\alpha) \geqq \frac{c}{2} q_{\left[x_{k}\right]} \quad\left(k \geqq k_{0}\right) .
$$

We shall carry the proof under the hypothesis that (3.26) holds. A similar argument applies if instead (3.26') is satisfied. Now, by Definition 3.1, there 
exists a positive number $\gamma$ less than $\pi$ and a sequence of numbers $\left\{\epsilon_{k}\right\}$ with $\left|\epsilon_{k}\right|=1$, such that

$$
\left|\arg \left\{\left[\alpha\left(u_{2}\right)-\alpha\left(u_{1}\right)\right] \epsilon_{k}\right\}\right| \leqq \gamma / 2 \quad(k=1,2, \cdots),
$$

for any $u_{1}$ and $u_{2}$ belonging to the interval $\left[x_{k}-d, x_{k}+d\right]\left(u_{1}<u_{2}\right)$. From (3.27) it will follow then that

$$
\operatorname{Re}\left\{\left[\alpha\left(u_{2}\right)-\alpha\left(u_{1}\right)\right] \epsilon_{k}\right\} \geqq \cos (\gamma / 2)\left|\alpha\left(u_{2}\right)-\alpha\left(u_{1}\right)\right| .
$$

In particular we shall get from (3.28) that the functions $\operatorname{Re}\left\{\left[\alpha\left(u+x_{k}\right)\right.\right.$ $\left.\left.-\alpha\left(x_{k}\right)\right] \epsilon_{k}\right\}$ and $\operatorname{Re}\left\{\left[\alpha\left(x_{k}\right)-\alpha\left(x_{k}-u\right)\right] \epsilon_{k}\right\}$ are nondecreasing functions of $u$ in the interval $[0, d)$. Also, dividing $\left[x_{k}, x_{k}+d\right]$ into subintervals, using (3.28), summing and taking the upper limit, one finds that

$$
\operatorname{Re}\left\{\left[\alpha\left(u+x_{k}\right)-\alpha\left(x_{k}\right)\right] \epsilon_{k}\right\} \geqq \cos (\gamma / 2) V_{x_{k}}^{x_{k}+u}(\alpha) \quad \text { for } 0 \leqq u \leqq d .
$$

In a similar manner one obtains

$$
\left(3.29^{\prime}\right) \quad \operatorname{Re}\left\{\left[\alpha\left(x_{k}\right)-\alpha\left(x_{k}-u\right)\right] \epsilon_{k}\right\} \geqq \cos (\gamma / 2) V_{x_{k}-u}^{x_{k}}(\alpha) \quad \text { for } 0 \leqq u \leqq d .
$$

Returning now to the limit function (3.25) it follows from Theorem 3.1 that it possesses the two representations (3.1) and (3.1'). The functions $\beta_{+}(u)$ and $\beta_{-}(u)$ of these representations can again be considered (taking if necessary a subsequence) to be given by

$$
\begin{aligned}
& \beta_{+}(u)=\lim _{k=\infty}\left(\frac{\alpha\left(x_{k}+u\right)-\alpha\left(x_{k}\right)}{q_{\left[x_{k}\right]}}\right) \text { and } \\
& \beta_{-}(u)=\lim _{k=\infty}\left(\frac{\alpha\left(x_{k}\right)-\alpha\left(x_{k}-u\right)}{q_{\left[x_{k}\right]}}\right) .
\end{aligned}
$$

Let now $\epsilon(|\epsilon|=1)$ be a limit point of the sequence $\left\{\epsilon_{k}\right\}$. It is readily seen from (3.28) and (3.30) that the functions $\operatorname{Re}\left\{\epsilon \beta_{+}(u)\right\}$ and $\operatorname{Re}\left\{\epsilon \beta_{-}(u)\right\}$ are nondecreasing in the interval $[0, d)$. They also vanish at the origin. Moreover, (3.30), (3.29), and (3.26) yield ( $k$ runs through a subsequence of the integers if necessary)

$$
\begin{aligned}
\operatorname{Re}\left\{\epsilon \beta_{+}(\eta)\right\} & =\lim _{k=\infty} \operatorname{Re}\left(\frac{\epsilon_{k}\left[\alpha\left(x_{k}+u\right)-\alpha\left(x_{k}\right)\right]}{q_{\left[x_{k}\right]}}\right) \\
& \geqq \cos (\gamma / 2) \lim \inf \left(\frac{V_{x_{k}}^{x_{k+\eta}}(\alpha)}{q_{\left[x_{k}\right]}}\right) \geqq(c / 2) \cos (\gamma / 2)>0 .
\end{aligned}
$$

As before we shall first establish that the above properties of $\beta_{+}(u)$ and $\beta_{-}(u)$ entail that $\phi(s)$ is not a constant. Indeed, if $\phi(s)$ were a constant then at all respective points of continuity one must have $\operatorname{Re}\left\{\epsilon \beta_{+}(u)\right\}=c_{+}$and $\operatorname{Re}\left\{\epsilon \beta_{-}(u)\right\}=c_{-}$where $c_{+}$and $c_{-}$are two non-negative constants. Using the 
monotonicity of $\operatorname{Re}\left\{\epsilon \beta_{+}(u)\right\}$ and $\operatorname{Re}\left\{\epsilon \beta_{-}(u)\right\}$ in the interval $(0, d)$, one finds that in this open interval one must have everywhere

$$
\operatorname{Re}\left\{\epsilon \beta_{+}(u)\right\} \equiv c_{+} \geqq 0 \text { and } \operatorname{Re}\left\{\epsilon \beta_{-}(u)\right\} \equiv c_{-} \geqq 0 \text {. }
$$

Recalling that $0<\eta<d$ we, furthermore, find (using (3.31) and (3.32)) that

$$
c_{+}=\operatorname{Re}\left\{\epsilon \beta_{+}(\eta)\right\} \geqq(c / 2) \cos (\gamma / 2)>0 .
$$

As $\beta_{ \pm}(0)=0,(3.1)$ and $\left(3.1^{\prime}\right)$ yield in our case

$$
\operatorname{Re}\{\epsilon \phi(s)\}=c_{+} \text {for } \sigma>0 \text {, and } \operatorname{Re}\{\epsilon \phi(s)\}=-c_{-} \text {for } \sigma<0 .
$$

This, clearly, leads to the contradiction $0<c_{+}=-c_{-} \leqq 0$. The nonconstancy of $\phi(s)$ is thus established.

The last step in the proof which consists in showing that $\phi(s)$ must have a singularity on the imaginary axis is exactly the same as the one given in the proof of the special case. One notes that in the contrary case $\phi(s)$ would be an entire function, which verifies a condition of the type (3.17). This leads easily to the conclusion that $\phi(s)$ must be a constant. But we have just shown that this is not the case. This completes the proof of the theorem.

We remark again that since any singular point of $\phi(s)$ is also a singularity of $f(s)$, we have obtained as a by product the following result:

COROLlaRY 3. Let $f(s)$ be represented by the Laplace transform (1.4) where $\alpha(u)$ belongs to the class $\Delta$, then $f(s)$ has at least one singular point on its axis of convergence.

4. Applications. The remainder of this paper will be devoted to applications of the previous results to complex variable Tauberians. In this section we shall give some "rough" Tauberians in which we shall have the relation $O$ instead of $\sim$. The more refined Tauberians of the type mentioned in the Introduction will be given in the last section. We point out, however, that as far as the relation $O$ is concerned the results of this section are rather precise. Also, the hypotheses underlying the theorems of this section are weaker than those needed for the more refined theorems. First we shall establish

THEOREM 4.1. Let $f(s)$ be represented in the half-plane $\operatorname{Re}\{s\}>0$ by (1.4) ( $\sigma=0$ being the abscissa of absolute convergence). Suppose that $\alpha(u)$ belongs to the class $\Omega$ and let

$$
\phi(s)=\sum_{n=0}^{\infty} a_{n} e^{-n s}
$$

be a Taylor $D$ series converging for $\operatorname{Re}\{s\}>0$, and such that $f(s)-\phi(s)$ is regular at the origin. Let, now, $\left\{q_{n}\right\}$ be a "regular" majorant of $\left\{a_{n}\right\}$. By this we shall always mean that $q_{n} \geqq\left|a_{n}\right|$ and that it satisfies the regularity conditions (2.18). Then 


$$
\alpha(u)=\sum_{n<u} a_{n}+c+O\left(q_{[u]}\right),
$$

where $c$ is a constant. In particular one has

$$
V_{u}^{u+1}(\alpha)=O\left(q_{[u]}\right) .
$$

Proof. Since $\alpha(u)$ belongs to $\Omega$ it is readily seen that

$$
\lim _{u^{\prime}=\infty}\left(\frac{\left|\alpha\left(u^{\prime}+1\right)-\alpha\left(u^{\prime}\right)\right|}{V_{u^{\prime}}^{u^{\prime}+1}(\alpha)}\right)=1,
$$

where $u^{\prime}$ runs through numbers for which $V_{u^{\prime}}^{u^{\prime}+1}(\alpha) \neq 0$. This shows that $\left(4.2^{\prime}\right)$ is a consequence of (4.2). Nevertheless in our proof we shall first establish $\left(4.2^{\prime}\right)$ and then deduce (4.2) from it. We also note that there is no loss of generality in establishing $\left(4.2^{\prime}\right)$ for $u$ running through the integers only. Now, let us put

$$
\begin{gathered}
V_{n}^{n+1}(\alpha)=b_{n}, \\
d_{n}=b_{n} / q_{n} .
\end{gathered}
$$

Then (since $\lim \sup b_{n}^{1 / n}=1$ and $\left.\lim q_{n}^{1 / n}=1\right)$ we have

$$
\lim \sup d_{n}^{1 / n}=1 \text {. }
$$

It is evident that $\left(4.2^{\prime}\right)$ is the same as stating that $\left\{d_{n}\right\}$ is bounded. We shall assume by way of contradiction that $\left(4.2^{\prime}\right)$ is false and that instead we have

$$
\lim \sup d_{n}=\infty \text {. }
$$

This, as we shall see, will lead to a contradiction.

Let $\log p_{n}$ be the smallest concave majorant of $\log d_{n}$ considered as a function of $n$. Using (4.6) and the minimal property of $\left\{p_{n}\right\}$, one sees that the last sequence has the following properties:

$$
\begin{aligned}
& \text { (i) } p_{n} \geqq d_{n}, \\
& \text { (ii) } \log p_{n+1}-2 \log p_{n}+\log p_{n-1} \leqq 0, \\
& \text { (iii) } p_{n+1} / p_{n} \downarrow 1, \\
& \text { (iv) } p_{n} \uparrow \infty, \\
& \text { (v) } p_{n_{k}}=d_{n_{k}} \text { for an infinite subsequence }\left\{n_{k}\right\} .
\end{aligned}
$$

We note that in establishing properties (iv) and (v) one uses (4.7). Now, put

$$
q_{n}^{\prime}=p_{n} q_{n} .
$$

Since both $\left\{p_{n}\right\}$ and $\left\{q_{n}\right\}$ have the regularity properties (2.19) their product $\left\{q_{n}^{\prime}\right\}$ will also have these properties. Furthermore, from (4.4), (4.5), (4.8), and (4.9) we get 
(4.10) $\quad q_{n}^{\prime} \geqq b_{n}=V_{n}^{n+1}(\alpha)$ for $n \geqq 0$, and $q_{n_{k}}^{\prime}=b_{n_{k}}=V_{n_{k}}^{n_{k}+1}(\alpha)$ for $k \geqq 1$. In other words, $\left\{n_{k}\right\}$ is a sequence of principal indices of $f(s)$ associated with the majorant $\left\{q_{n}^{\prime}\right\}$. Let

$$
h(s)=f(s)-\phi(s)
$$

Then

$$
h(s)=\int_{0}^{\infty} e^{-s u} d \alpha^{*}(u) \quad \text { for } \operatorname{Re}\{s\}>0,
$$

where

$$
\alpha^{*}(u)=\alpha(u)-\sum_{n<u} a_{n} .
$$

Now, from (4.12'), (4.10), and (4.4), we get

$$
V_{n}^{n+1}\left(\alpha^{*}\right) \leqq V_{n}^{n+1}(\alpha)+\left|a_{n}\right| \leqq q_{n}^{\prime}+q_{n} \leqq 2 q_{n}^{\prime} \quad \text { for } n \geqq n_{0} .
$$

Thus, we can apply Theorem 2.2 to the family

$$
h_{n}(s)=\frac{h(s)-\int_{0}^{n} e^{-s u} d \alpha^{*}(u)}{q_{n}^{\prime} e^{-n s}},
$$

so that $\left\{h_{n}(s)\right\}$ is uniformly bounded in any compact region where $h(s)$ is regular. In particular this will be true for a certain neighborhood of the origin. Let, now, $\phi(s)$ be any limit function of $\left\{h_{n_{k}}(s)\right\}$ where $\left\{n_{k}\right\}$ is the sequence of principal indices satisfying (4.10). Applying Theorem 3.1 it follows that $\phi(s)$ is analytic in a domain composed of the half-plane $\operatorname{Re}\{s\}$ $>0$, the half-plane $\operatorname{Re}\{s\}<0$, and a certain neighborhood of the origin. It will also possess the representations

$$
\begin{array}{ll}
\phi(s)=\int_{0}^{\infty} e^{-s u} d \beta_{+}(u) & \text { for } \operatorname{Re}\{s\}>0, \\
\phi(s)=-\int_{0}^{\infty} e^{+s u} d \beta_{-}(u) & \text { for } \operatorname{Re}\{s\}<0,
\end{array}
$$

where

$$
\begin{aligned}
& \beta_{+}(u)=\lim _{k^{\prime}=\infty}\left(\frac{\alpha^{*}\left(u+n_{k^{\prime}}\right)-\alpha^{*}\left(n_{k^{\prime}}\right)}{q_{n_{k^{\prime}}^{\prime}}^{\prime}}\right), \\
& \beta_{-}(u)=\lim _{k^{\prime}=\infty}\left(\frac{\alpha^{*}\left(n_{k^{\prime}}\right)-\alpha^{*}\left(n_{k^{\prime}}-u\right)}{q_{n_{k^{\prime}}^{\prime}}^{\prime}}\right)
\end{aligned}
$$


( $\left\{k^{\prime}\right\}$ is a certain subsequence of the integers). But, one sees that for a fixed $u$

$$
\frac{1}{q_{n}{ }^{\prime}} \sum_{|k|<u}\left|a_{n+k}\right| \leqq \frac{1}{p_{n} q_{n}} \sum_{|k|<u} q_{n+k} \rightarrow 0 \quad(n \rightarrow \infty) .
$$

(This results from the fact that $\left(\sum_{|k|<u} q_{n+k}\right) / q_{n}=O(1)$ and $p_{n} \uparrow \infty_{\text {.) }}$ Since, now, by $\left(4.12^{\prime}\right)$,

$$
\begin{aligned}
& \frac{\alpha^{*}(u+n)-\alpha^{*}(n)}{q_{n}{ }^{\prime}}=\frac{\alpha(u+n)-\alpha(n)}{q_{n}{ }^{\prime}}-\frac{\sum_{n+u>v \geqq n} a_{\nu}}{q_{n}{ }^{\prime}}, \\
& \frac{\alpha^{*}(n)-\alpha^{*}(n-u)}{q_{n}{ }^{\prime}}=\frac{\alpha(n)-\alpha(n-u)}{q_{n}{ }^{\prime}}-\frac{\sum_{n>v \geqq n-u} a_{\nu}}{q_{n}{ }^{\prime}},
\end{aligned}
$$

it follows from (4.17) and (4.16) that (4.15) can be replaced by

$$
\begin{aligned}
& \beta_{+}(u)=\lim _{\boldsymbol{k}^{\prime}=\infty}\left(\frac{\alpha\left(n_{k^{\prime}}+u\right)-\alpha\left(n_{k^{\prime}}\right)}{q_{n_{k^{\prime}}}^{\prime}}\right), \\
& \beta_{-}(u)=\lim _{\boldsymbol{k}^{\prime}=\infty}\left(\frac{\alpha\left(n_{k^{\prime}}\right)-\alpha\left(n_{k^{\prime}}-u\right)}{q_{n_{k^{\prime}}^{\prime}}^{\prime}}\right) .
\end{aligned}
$$

Let us also note that (4.3), (4.4), (4.10), and (4.18) imply that

$$
\left|\beta_{+}(1)\right|=1 \text {. }
$$

Now it is easily seen from the fact that $\alpha(u)$ belongs to the class $\Omega$ and from (4.18) that $\beta_{+}(u)=\epsilon \gamma_{+}(u)$ and $\beta_{-}(u)=\epsilon \gamma_{-}(u)$ where $\gamma_{+}(u)$ and $\gamma_{-}(u)$ are nondecreasing functions $\left(\gamma_{ \pm}(0)=0\right)$ and $\epsilon$ is a constant of modulus 1 . Thus, for the function $\phi_{1}(s)=\epsilon^{-1} \phi(s)$ we have the representations:

$$
\begin{array}{lr}
\phi_{1}(s)=\int_{0}^{\infty} e^{-s u} d \gamma_{+}(u) & \text { for } \operatorname{Re}\{s\}>0 \text { and } \\
\phi_{1}(s)=-\int_{0}^{\infty} e^{+s u} d \gamma_{-}(u) & \text { for } \operatorname{Re}\{s\}<0 .
\end{array}
$$

But we find (as in the proof of the special case of Theorem 3.2) that

$$
\limsup _{\sigma=+0} \phi_{1}(\sigma) \geqq \int_{0}^{1} d \gamma_{+}(u)=\gamma_{+}(1)=1>0
$$

and that

$$
\limsup _{\sigma=-0} \phi_{1}(\sigma)=-\limsup _{\sigma=-0} \int_{0}^{\infty} e^{-s u} d \gamma_{-}(u) \leqq 0 .
$$

This shows that $\phi_{1}(s)$ is not even continuous at the origin, and furnishes a 
contradiction to our previous assertion that $\phi_{1}(s)$ is regular there. This completes the proof of $\left(4.2^{\prime}\right)$.

We shall now establish (4.2). To this end put

$$
g_{x}(s)=\frac{f(s)-\phi(s)-\left[\int_{0}^{x} e^{-s u} d \alpha(u)-\sum_{n<x} a_{n} e^{-n s}\right]}{q_{[x]} e^{-x s}} .
$$

Since (4.2') holds we may use Theorem 2.2 and conclude that the family $\left\{g_{x}(s)\right\}$ is bounded in any point where $h(s)=f(s)-\phi(s)$ is regular. In particular this will be true for $s=0$. Thus, we find from (4.20) that

$$
g_{x}(0)=\frac{h(0)-\left[\alpha(x)-\alpha(0)-\sum_{n<x} a_{n}\right]}{q_{[x]}}=O(1) \quad(x \rightarrow \infty) .
$$

But this is nothing else but (4.2) if we let $c=h(0)+\alpha(0)$. The theorem is now completely established.

One may say that under the conditions of Theorem 4.1 the knowledge of the singularity at the origin suffices in determining the growth of $\alpha(u+h)$ $-\alpha(u)$. This is due to the fact that the assumption that $\alpha(u)$ belongs to the class $\Omega$ ensures the origin to be as "strong" a singularity as any other on the imaginary axis. We shall now prove another variant of Theorem 4.1 in which $\alpha(u)$ will belong only to the class $\Delta$. In this case, however, we shall have to consider all the singularities of $f(s)$ on the imaginary axis.

THEOREM 4.2. Let $f(s)$ be represented in the half-plane $\operatorname{Re}\{s\}>0$ by (1.4) ( $\sigma=0$ being the abcissa of absolute convergence). Suppose that $\alpha(u)$ belongs to the class $\Delta$. Let $\left\{q_{n}\right\}$ be a regular sequence satisfying (2.18) and suppose that the following holds: one can associate with each singular point $s=i \tau$ of $f(s)$ on the imaginary axis a Taylor $-D$ series $\phi_{\tau}(s)=\sum a_{n}^{\tau} \exp \left(-n h_{\tau} s\right)\left(h_{\tau}\right.$ being a positive constant) such that $f(s)-\phi_{\tau}(s)$ is regular at the point ir and

$$
a_{n}^{\tau}=O\left(q_{\left[n h_{\tau}\right]}\right)
$$

Then

$$
V_{u}^{u+1}(\alpha)=O\left(q_{[u]}\right)
$$$$
\text { as } u \rightarrow \infty \text {. }
$$

Proof. Applying if necessary a change of variable $s=s^{\prime} l, u^{\prime}=u e$, one may assume that the constant $d$ associated with $\alpha(u)$ in Definition 3.1 is greater than 1. It is also enough to establish (4.22) when $u$ runs through the integers. We shall follow the proof of Theorem 4.1 and define again the sequences $\left\{b_{n}\right\}$ and $\left\{d_{n}\right\}$ by (4.4) and (4.5). ( $\left\{d_{n}\right\}$ will thus verify (4.6).) The theorem then amounts to showing that $\left\{d_{n}\right\}$ is bounded. As before we shall show that the 
contrary assumption (namely that (4.7) holds) leads to a contradiction. Let again $\left\{\log p_{n}\right\}$ be the smallest concave majorant of $\left\{\log d_{n}\right\}$. (The sequence $\left\{p_{n}\right\}$ will satisfy (4.8).) Finally, let $q_{n}^{\prime}=q_{n} p_{n}$. The sequence $\left\{q_{n}^{\prime}\right\}$ will possess properties (4.10). Let, now, $s_{0}=i \tau_{0}$ be any singular point of $f(s)$ on the imaginary axis. (By Corollary 3 at the end of $\$ 3$ we know that at least one such singularity exists.) Let $\phi_{r_{0}}(s)$ be the corresponding Taylor series associated with the singularity. Put $h_{\tau_{0}}(s)=f(s)-\phi_{\tau_{0}}(s)$. Then, clearly, we have

$$
h_{\tau_{0}}(s)=\int_{0}^{\infty} e^{-s u} d \alpha_{\tau_{0}}(u) \quad \text { for } \operatorname{Re}\{\mathrm{s}\}>0,
$$

where

$$
\alpha_{\tau_{0}}(u)=\alpha(u)-\sum_{n h_{\tau_{0}}<u} a_{n}^{\tau_{0}} .
$$

From (4.10), (4.21), and (4.24) we get

$$
V_{n}^{n+1}\left(\alpha_{\tau_{0}}\right) \leqq V_{n}^{n+1}(\alpha)+\sum_{n \leqq k h_{\tau_{0}}<n+1}\left|a_{k}^{\tau_{0}}\right| \leqq q_{n}^{\prime}+O\left(q_{n}\right)<2 q_{n}^{\prime} \quad\left(n \geqq n_{0}\right) .
$$

Hence, by Theorem 2.2 the family

$$
h_{n}^{\tau_{0}}(s)=\frac{h_{\tau_{0}}(s)-\int_{0}^{n} e^{-s u} d \alpha_{\tau_{0}}(u)}{q_{n}^{\prime} e^{-n s}}
$$

is uniformly bounded in any compact region where $h_{\tau_{0}}(s)$ is analytic. Let $\phi_{\tau_{0}}(s)$ be any limit function of the subsequence $\left\{h_{n_{k}}^{\tau_{0}}(s)\right\}$ where $\left\{n_{k}\right\}$ is the sequence of principal indices associated with $\left\{q_{n}^{\prime}\right\}$ in (4.10). By Theorem 3.2 it follows that $\phi_{\tau_{0}}(s)$ is analytic in a region composed of the half-planes $\operatorname{Re}\{s\}>0$ and $\operatorname{Re}\{s\}<0$ and a certain neighborhood of the point $i \tau_{0}$. It also has the representations:

$$
\begin{array}{ll}
\phi_{\tau_{0}}(s)=\int_{0}^{\infty} e^{-s u} d \beta_{+}^{\tau_{0}}(u) & \text { for } \operatorname{Re}\{s\}>0, \\
\phi_{\tau_{0}}(s)=-\int_{0}^{\infty} e^{s u} d \beta_{-}^{\tau_{0}}(u) & \text { for } \operatorname{Re}\{s\}<0 .
\end{array}
$$

There exists, furthermore, a subsequence of the integers $\left\{k^{\prime}\right\}$ such that

$$
\begin{aligned}
& \beta_{+}^{\tau_{0}}(u)=\lim _{k^{\prime}=\infty}\left(\frac{\alpha_{\tau_{0}}\left(n_{k^{\prime}}+u\right)-\alpha_{\tau_{0}}\left(n_{k^{\prime}}\right)}{q_{n_{k^{\prime}}}^{\prime}}\right), \\
& \beta_{-}^{\tau_{0}}(u)=\lim _{k^{\prime}=\infty}\left(\frac{\alpha_{\tau_{0}}\left(n_{k^{\prime}}\right)-\alpha_{\tau_{0}}\left(n_{k^{\prime}}-u\right)}{q_{n_{k^{\prime}}^{\prime}}^{\prime}}\right) .
\end{aligned}
$$


Now it is easy to see (in exactly the same manner as (4.16) was established) that for a fixed $u$

$$
\frac{1}{q_{n}^{\prime}} \sum_{\left|k h_{\tau_{0}}-n\right|<u}\left|a_{k}^{\tau_{0}}\right|=o(1) \quad(n \rightarrow \infty) .
$$

From (4.24), (4.27), and (4.28) it will follow now that $\beta_{+}^{\tau_{0}}(u)$ and $\beta_{-}^{\tau_{0}}(u)$ are actually independent of $\tau_{0}$ since we may replace (4.27) by

$$
\begin{aligned}
& \beta_{+}^{\tau_{0}}(u)=\lim _{k^{\prime}=\infty}\left(\frac{\alpha\left(n_{k^{\prime}}+u\right)-\alpha\left(n_{k^{\prime}}\right)}{q_{n_{k^{\prime}}}^{\prime}}\right), \\
& \beta_{-}^{\tau_{0}}(u)=\lim _{k^{\prime}=\infty}\left(\frac{\alpha\left(n_{k^{\prime}}\right)-\alpha\left(n_{k^{\prime}}-u\right)}{q_{n_{k^{\prime}}}^{\prime}}\right) .
\end{aligned}
$$

Letting $\beta_{+}^{\tau_{0}}(u)=\beta_{+}(u), \beta_{-}^{\tau_{0}}(u)=\beta_{-}(u)$, and $\phi^{\tau_{0}}(s)=\phi(s)$, we may rewrite (4.26) as

$$
\begin{array}{ll}
\phi(s)=\int_{0}^{\infty} e^{-s u} d \beta_{+}(u) & \text { for } \operatorname{Re}\{s\}>0, \\
\phi(s)=-\int_{0}^{\infty} e^{s u} d \beta_{-}(u) & \text { for } \operatorname{Re}\{s\}<0 .
\end{array}
$$

Let us further note that one sees from (4.29) and (4.30) that $\phi(s)$ is also a limit function of the family

$$
f_{n_{k}}(s)=\frac{f(s)-\int_{0}^{n_{k}} e^{-s u} d \alpha(u)}{q_{n_{k}}^{\prime} e^{-n_{k s}}} .
$$

This implies that $\phi(s)$ is regular at all points of regularity of $f(s)$ on the imaginary axis. Also, by way of construction $\phi(s)$ is regular at the chosen point $i \tau_{0}$ (which is a singularity of $f(s)$ ). However, since $\phi(s)$ turned out to be really independent of $i \tau_{0}$ it follows easily that $\phi(s)$ is regular also at any other point $i \tau$. (We have only to define the corresponding family $h_{n_{k^{\prime}}}^{\tau}(s)$ where $\left\{n_{k^{\prime}}\right\}$ is the sequence of (4.29) and pick any limit function $\phi_{r}(s)$. This limit function is readily seen to be $\phi(s)$ and is regular at $i \tau$.) Summing the above results we conclude that $\phi(s)$ is an entire function. Now, as we have already pointed out $\phi(s)$ is a limit function of the family $\left\{f_{n_{k}}(s)\right\}$ defined by (4.31). Also, because of (4.10), $\left\{n_{k}\right\}$ is a sequence of principal indices associated with $\left\{q_{n}^{\prime}\right\}$. Assume first that the imaginary axis is not a natural boundary for $f(s)$. One may then apply Theorem 3.2 to the family $\left\{f_{n_{k}}(s)\right\}$ and deduce that $\phi(s)$ must possess a singularity on the imaginary axis. This, clearly, contradicts the conclusions just obtained and establishes the theorem in this case. Furthermore, the assumption that $f(s)$ may be continued analytically beyond the half-plane $\operatorname{Re}\{s\}>0$ is really not necessary in this case, since, in 
the proof of Theorem 3.2, this assumption was used only to establish the fact that $\phi(s)$ can be continued analytically into the half-plane $\operatorname{Re}\{s\}<0$ where it possesses the representation (4.30). But these facts were already established in our case. Hence, the same method of proof as employed in the proof of Theorem 3.2 will lead to the conclusion that $\phi(s)$ must possess at least one singular point on the imaginary axis. This is in contradiction with our previous conclusions and the theorem is thus completely proved.

We shall now state a third theorem of the same type as the preceding two. It will concern Dirichlet series

$$
f(s)=\sum_{n=0}^{\infty} a_{n} e^{-\lambda_{n} \varepsilon},
$$

with exponents $0 \leqq \lambda_{n} \uparrow \infty$ and such that

$$
\lim \inf \left(\lambda_{n+1}-\lambda_{n}\right)=l>0 .
$$

This theorem will establish a rather precise estimate for the coefficients $\left\{a_{n}\right\}$ once the character of the singularities of $f(s)$ on a segment of the axis of convergence of length greater than $2 \pi / l$ is known.

THEOREM 4.3. Let $f(s)$ be represented in the half-plane $\operatorname{Re}\{s\}>0$ by (4.32) where the exponents satisfy $\left(4.32^{\prime}\right)(\sigma=0$ being the abcissa of absolute convergence). Let

$$
\phi(s)=\sum_{n=0}^{\infty} b_{n} e^{-c n s}
$$

be a Taylor-D series converging for $\operatorname{Re}\{s\}>0$ and such that $h(s)=f(s)-\phi(s)$ is regular on a segment of the imaginary axis of length greater than $2 \pi / l$. Let $\left\{q_{n}\right\}$ be a regular majorant of the $b_{n}$ 's. (That is, $q_{n} \geqq\left|b_{n}\right|$ and $\left\{q_{n}\right\}$ has the properties of (2.18).) Then

$$
a_{n}=O\left(q_{\left[\lambda_{n} / c\right]}\right) \quad \text { as } n \rightarrow \infty .
$$

The proof of Theorem 4.3 follows closely the lines of proof of the previous two theorems and shall therefore be omitted. We shall only remark that in the proof of Theorem 4.1 the contradiction obtained depended essentially on Landau's Theorem that the real point on the axis of convergence of (1.4) when $\alpha(u)$ is nondecreasing is a singularity. Similarly, in the proof of Theorem 4.2 we have used essentially Corollary 3 at the end of $\$ 3$. The corresponding result which one uses in the proof of Theorem 4.3 is a well known theorem due to Pólya which states that if $f(s)$ is represented by (4.32) with $\lambda_{n}$ 's satisfying $\left(4.32^{\prime}\right)$, then any segment of the axis of convergence of length greater than $2 \pi / l$ must contain a singularity of $f(s)$.

To show that the three results obtained in this section are rather precise let us apply them in a very special case, namely: when it is known that the 
relevant singularities are poles. (Other more general results can be easily formulated, e.g., if the singularities are algebrico-logarithmic.)

(a) Assume that $f(s)$ is represented by (1.4) and that $\alpha(u)$ belongs to the class $\Omega$. Assume, furthermore, that the origin is a pole of order $k$ so that $f(s)=A / s^{k}+A_{1} / s^{k-1}+\cdots+A_{k-1} / s+f_{1}(s)$ where $f_{1}(s)$ is regular at the origin. Letting

$$
\begin{aligned}
\phi(s) & =\frac{(-1)^{k-1} A}{(k-1) !}\left(\frac{1}{1-e^{-s}}\right)^{(k-1)}+\cdots+\frac{A_{k-1}}{1-e^{-s}} \\
& =\sum_{n=0}^{\infty}\left(\frac{A}{(k-1) !} n^{k-1}+\cdots+A_{k-1}\right) e^{-n s} \quad \text { for } \operatorname{Re}\{\mathrm{s}\}>0,
\end{aligned}
$$

one sees easily that $f(s)-\phi(s)$ is regular at the origin and that Theorem 4.1 may be applied. The result then takes the form

$$
\alpha(u)=[A /(k-1) !] n^{k}+O\left(n^{k-1}\right) .
$$

(b) Suppose that in (a) it is known only that $\alpha(u)$ belongs to the class $\Delta$. On the other hand suppose that it is known that all the singularities of $f(s)$ on the imaginary axis are poles of bounded order $k$. Then, in a similar fashion to the previous case, one constructs the Taylor- $D$ series $\phi_{r}(s)$ such that $f(s)-\phi_{\tau}(s)$ is regular at $i \tau$ and, applying Theorem 4.2, one finds that

$$
V_{u}^{u+1}(\alpha)=O\left(u^{k-1}\right) .
$$

(c) Let $f(s)$ be a Dirichlet series (4.32) with exponents satisfying (4.32'). Suppose that the only singularities of $f(s)$ on a segment $I$ of the imaginary axis (the axis of convergence) of length greater than $2 \pi / l$ are poles the greatest order of which is $k$. Then it is again a simple matter to construct a Taylor- $D$ series $\phi(s)=\sum b_{n} \exp (-n c s)$ having only poles on the imaginary axis of maximum order $k$ and such that $f(s)-\phi(s)$ will be regular on $I$. Applying Theorem 4.3, using the fact that $b_{n}=O\left(n^{k-1}\right)$, we find that

$$
a_{n}=O\left(\lambda_{n}^{k-1}\right) \text {. }
$$

5. Two lemmas. In this section we shall establish two lemmas which we shall need later and which are of interest by themselves.

LEMMA 5.1. Let

$$
f(z)=\sum_{n=0}^{\infty} a_{n} z^{n}
$$

be a Taylor series converging for $|z|<1$ and such that the point $z=1$ is its only singularity on the unit circle. Suppose, furthermore, that the sequence of moduli of the coefficients satisfies the following condition of regularity 


$$
\delta q_{n} \leqq\left|a_{n}\right| \leqq q_{n}
$$$$
(n=0,1, \cdots),
$$

where $\left\{q_{n}\right\}$ is a sequence having the properties (2.18) and where $\delta$ is a positive constant. Then

$$
\lim _{n=\infty}\left(a_{n} / a_{n+1}\right)=1 .
$$

Thus, we note, Lemma 5.1 is a kind of converse of a theorem of Fabry which asserts that (5.1) and (5.3) entail that $z=1$ is a singularity. It is well known that the converse of Fabry's theorem does not hold even if $z=1$ is the only singularity on the unit circle. Yet the above lemma asserts that the converse does hold if the sequence of moduli of the coefficients is regular enough. Now Lemma 5.1 in a more general form was established in [2, p. 506]. We shall give here a different and simpler proof of the lemma which uses the methods of this paper.

Proof of Lemma 5.1. Assume by way of contradiction that the lemma is false. Since (5.2) implies that $a_{n+1} / a_{n}=O(1)$ it will follow that there exists a sequence of integers $\left\{n_{k}\right\}$ such that

$$
\lim _{k=\infty}\left(a_{n_{k}+1} / a_{n_{k}}\right)=l \neq 1 .
$$

Consider now the family

$$
f_{n}(z)=\frac{f(z)-\sum_{\nu=0}^{n-1} a_{\nu} z^{\nu}}{a_{n} z^{n}}=1+\left(a_{n+1} / a_{n}\right) z+\left(a_{n+2} / a_{n}\right) z^{2}+\cdots
$$

for $|z|<1$. It follows from (5.2) and Theorem 2.2 (formulated in the obvious way for Taylor series) that $\left\{f_{n}(z)\right\}$ is uniformly bounded in any compact region where $f(z)$ is analytic. Let $g(z)$ be a limit function of the subsequence $\left\{f_{n_{k}}(z)\right\}$ where $\left\{n_{k}\right\}$ is the corresponding sequence of (5.4). There is no loss of generality in assuming that we already have

$$
g(z)=\lim _{z=\infty} f_{n_{k}}(z) \text {. }
$$

Then by Theorem 3.1 (again formulated for Taylor series) we shall have the two representations

$$
\begin{array}{ll}
g(z)=1+b_{1} z+b_{2} z^{2}+\cdots & \text { for }|z|<1, \\
g(z)=-\left(\frac{b_{-1}}{z}+\frac{b_{-2}}{z^{2}}+\cdots\right) & \text { for }|z|>1,
\end{array}
$$

where

$$
b_{\nu}=\lim _{\boldsymbol{x}=\infty}\left(\frac{a_{n_{k}+\nu}}{a_{n_{k}}}\right) \quad \text { for } \nu= \pm 1, \pm 2, \cdots
$$


Furthermore, using Theorem 3.1 and our hypotheses we find that $g(z)$ can possess only one singularity, $z=1$, in the whole closed plane. Moreover, the singularity can be only a simple pole. Thus $g(z)$ must be a linear function which is determined uniquely by the fact that $g(0)=1$ and $g(\infty)=0$. We have:

$$
g(z)=\frac{1}{1-z}=1+z+z^{2}+\cdots \quad \text { for }|z|<1 .
$$

Comparing the last result with (5.7) one finds that $b_{1}=1$. On the other hand from (5.4) and (5.8) one gets that $b_{1}=l \neq 1$. This yields a contradiction and the proof of the lemma is complete.

We pass now to the second lemma which deals with the existence of Laplace transforms with a prescribed single singularity on the axis of convergence.

LEMMA 5.2. Let

$$
g(s)=\sum_{n=0}^{\infty} a_{n} e^{-n s}
$$

be a Taylor-D series converging for $\operatorname{Re}\{s\}>0$ and having the following properties: (i) The $a_{n}$ 's are regular enough. More precisely they verify (5.2). (ii) The only singularities of $f(s)$ on the imaginary axis are the points $2 k \pi i$ with $k$ $=0, \pm 1, \cdots$. Then there exists an analytic function $\phi(s)$ which is represented in the half-plane $\operatorname{Re}\{s\}>0$ by

$$
\phi(s)=\int_{0}^{\infty} e^{-s u} \psi(u) d u,
$$

and which has the following properties:

(a) $s=0$ is the only singularity of $\phi(s)$ on the imaginary axis.

(b) $g(s)-\phi(s)$ is regular at the origin.

(c) $\psi(u)$ is an entire function of exponential type such that

$$
\psi(u) \sim a_{[u]}
$$

as $u \rightarrow \infty$.

Proof. Let $T$ be a number such that $0<T<\pi$ and let us pick a positive number $\delta_{0}$ such that $g(s)$ is regular on the two segments $-\delta_{0} \leqq \sigma \leqq 0, t=T$ and $-\delta_{0} \leqq \sigma \leqq 0, t=-T$. We shall denote by $C_{\epsilon}(\epsilon>0)$ the directed polygonal line composed of the three segments $-\delta_{0} \leqq \sigma \leqq \epsilon, t=-T ; \sigma=\epsilon,-T \leqq t \leqq T$; and $\epsilon \geqq \sigma \geqq-\delta_{0}, t=T$ (the direction being from $-\delta_{0}-i T$ to $-\delta_{0}+i T$ ). Then, we claim, the function

$$
\phi(s)=\frac{1}{2 \pi i} \int_{L_{\epsilon}} \frac{g(\zeta)}{s-\zeta} d \zeta \quad(\operatorname{Re}\{s\}>\epsilon)
$$

has all the desired properties. Indeed, if we deform the line $L_{\epsilon}$ continuously 
so that it never sweeps a singularity of $g(s)$ and so that its end points remain fixed, we obtain (by the corresponding integral (5.11)) the analytic continuation of $\phi(s)$ to the region which is the whole plane cut along the line integration. Since, however, we may by the above deformation deform $L_{\epsilon}$ into a curve $L$ such that all its points which lie in the half-plane $\operatorname{Re}\{s\}>0$ are to be found in an arbitrary neighborhood of the origin, we conclude that $\phi(s)$ is analytic in $\operatorname{Re}\{s\} \geqq 0$ except for the origin. This establishes property (a). Now, to deduce (b), let $s=\sigma+i t$ be a point in the rectangle $\epsilon<\sigma<\delta$, $|t|<T$. By Cauchy's theorem

$$
g(s)=\frac{1}{2 \pi i} \int_{L_{\delta}} \frac{g(\zeta)}{\zeta-s} d \zeta+\frac{1}{2 \pi i} \int_{L_{\epsilon}} \frac{g(\zeta)}{s-\zeta} d \zeta=g_{1}(s)+\phi(s) .
$$

But it is readily seen that $g_{1}(s)$ is regular at the origin. Since $g(s)-\phi(s)=g_{1}(s)$, property (b) is also established. Finally, in order to deduce (c) let us note that if $\operatorname{Re}\{\zeta-s\}<0$, we may write

$$
\frac{1}{s-\zeta}=\int_{0}^{\infty} e^{(\zeta-s) u} d u
$$

which when substituted in (5.11) yields

$$
\begin{aligned}
\phi(s) & =\frac{1}{2 \pi i} \int_{L_{\epsilon}} \frac{g(\zeta)}{s-\zeta} d \zeta=\frac{1}{2 \pi i} \int_{L_{\epsilon}} g(\zeta) d \zeta \int_{0}^{\infty} e^{(\zeta-s) u} d u \\
& =\int_{0}^{\infty} e^{-s u} \psi(u) d u
\end{aligned}
$$

where

$$
\psi(u)=\frac{1}{2 \pi i} \int_{L_{\epsilon}} g(\zeta) e^{\zeta u} d \zeta .
$$

This shows that indeed $\phi(s)$ is represented in the half-plane $\operatorname{Re}\{s\}>0$ by (5.10) and that $\psi(u)$ is of exponential type. It is left only to establish the asymptotic relation given in (c). To this end we shall first note that $\psi(u)$ which is given by $\left(5.13^{\prime}\right)$ is really independent of $\epsilon$. Also, if we write $\zeta=\xi+i \eta$ and $u=v+i w$, we get from $\left(5.13^{\prime}\right)$ for $u$ in the half-plane $v \geqq 0$ :

$$
|\psi(u)| \leqq C_{\epsilon} e^{e v+T|W|} \quad\left(C_{\epsilon} \text { being a constant }\right) .
$$

Since $\psi(u)$ is independent of $\epsilon$, this shows that

$$
\limsup _{r=\infty}\left(\frac{\log \left|\psi\left(r e^{i \theta}\right)\right|}{r}\right) \leqq T|\sin \theta| \quad \text { for } \mid \theta 1<\pi / 2 .
$$

Now we claim that 


$$
\psi(n)=\left(\frac{1}{2 \pi i} \int_{L_{\epsilon}} g(\zeta) e^{n \zeta} d \zeta\right)=a_{n}+O\left(e^{-\delta n}\right)=\left(a_{n}+o\left(a_{n}\right)\right) \text { as } n \rightarrow \infty,
$$

for some positive constant $\delta$. Indeed, from (5.9) and the residue theorem we have for $s=\sigma+i t$ with $\epsilon<\sigma<1$ and $|t|<T<\pi$ :

$$
\begin{aligned}
g(s)=\sum_{n=0}^{\infty} a_{n} e^{-n s} & =\frac{1}{2 \pi i} \int_{L_{\epsilon}-L_{1}} \frac{g(\zeta)}{1-e^{(\zeta-s)}} d \zeta \\
& =\frac{1}{2 \pi i} \int_{L_{\epsilon}} \frac{g(\zeta)}{1-e^{(\zeta-s)}} d \zeta-\frac{1}{2 \pi i} \int_{L_{1}} \frac{g(\zeta)}{1-e^{(\zeta-s)}} d \zeta \\
& =\phi_{1}(s)-h(s) .
\end{aligned}
$$

In the half-plane $\operatorname{Re}\{s\}>\epsilon, \phi_{1}(s)$ can be expanded in a Taylor- $D$ series

$$
\phi_{1}(s)=\frac{1}{2 \pi i} \int_{L_{e}} g(\zeta) \sum_{0}^{\infty} e^{-n(s-\zeta)}=\sum_{0}^{\infty} \psi(n) e^{-n s} .
$$

Consequently we get in the same half-plane

$$
h(s)=\phi_{1}(s)-g(s)=\sum_{0}^{\infty}\left(\psi(n)-a_{n}\right) e^{-n s} .
$$

But, using the integral representation of $h(s)$ one sees easily (by deforming the line of integration) that $h(s)$ is analytic in the half-plane $\operatorname{Re}\{s\}>0$ and also on a segment of the imaginary axis of length greater than $2 \pi$. This shows that the series (5.17) must converge at a point $s=-\delta<0$ from which our assertion (5.15) follows. We shall now make use of a theorem proved in [2, pp. 498 and 503] from which it will follow (on account of (5.2), (5.14), (5.15), and $T<\pi)$ that

(i) $\psi(u)=O\left(a_{[u]}\right)$ for $u \rightarrow \infty$.

(ii) The family

$$
\psi_{n}(z)=\psi(n+z) / \psi(n) \quad(n=0,1, \cdots)
$$

is a normal family in any bounded region of the plane.

(iii) Any limit function $\phi(z)$ of the above family satisfies an inequality of the type

$$
|\phi(x+i y)| \leqq A e^{T^{\prime}|y|} \quad \text { where } T<T^{\prime}<\pi .
$$

Now in our case we shall show that all limit functions reduce to the constant 1 . This will give that $\lim \psi_{n}(z)=1(n \rightarrow \infty)$ which in turn will yield the desired asymptotic formula since, using (5.15), we shall get

$$
1=\lim [\psi(u) / \psi([u])]=\lim \left[\psi(u) / a_{[u]}\right],
$$

which is the desired relation. Indeed, if $\phi(z)$ is any limit function and $m$ is any integer, we have 


$$
\phi(m)=\lim \left(\frac{\psi(n+m)}{\psi(n)}\right)=\lim \left(\frac{a_{n+m}}{a_{n}}\right)=1 .
$$

(We use here (5.15) and Lemma 5.1.) Thus $\phi(z)-1$ is an entire function which vanishes at all the integers and which, furthermore, satisfies (5.18) with $T^{\prime}<\pi$. By a well known theorem due to Carlson it follows that $\phi(z)-1 \equiv 0$. This, as we have just shown, proves the lemma.

6. Tauberians involving lower estimates. In $\$ 4$ we have discussed a number of Tauberians which gave an upper estimate to $V_{u}^{u+1}(\alpha)=\int_{u}^{u+1}|d \alpha(u)|$ in terms of a certain majorant of the sequence of coefficients of a related Taylor- $D$ series. We shall now show that under certain additional conditions a similar lower bound can be derived. We shall establish three results which correspond to the three theorems of $\$ 4$.

THEOREM 6.1. Under the conditions of Theorem 4.1 suppose that the onesided inequality $\left|a_{n}\right| \leqq q_{n}$ may be replaced by the two-sided inequality (5.2) and that, furthermore, $\lim \left(a_{n} / a_{n+1}\right)=1$. Then there exist positive numbers $N$ and C such that

$$
\frac{1}{C}\left|a_{[u]}\right| \leqq \int_{u}^{u+N}|d \alpha(u)| \leqq C\left|a_{[u]}\right| \quad \text { for } u \text { sufficiently large. }
$$

Proof. By Theorem 4.1 and (5.2) there exists a constant $B$ such that

$$
\left|\alpha(u)-\sum_{n<u} a_{n}\right| \leqq B\left|a_{[u]}\right| .
$$

Also, since $\alpha(u)$ belongs to the class $\Omega$ and $a_{n} / a_{n+1} \rightarrow 1$, one has for a fixed $N$

$$
V_{u}^{u+N}(\alpha) \sim|\alpha(u+N)-\alpha(u)| \text { and }\left|\sum_{u \leqq k<u+N} a_{k}\right| \sim N\left|a_{[u]}\right| .
$$

Thus

$$
\begin{aligned}
V_{u}^{u+N}(\alpha)= & (1+o(1))|\alpha(u+N)-\alpha(u)| \geqq(1-o(1))\left|\sum_{u \leqq k<u+N} a_{k}\right|, \\
& -2 B(1+o(1))\left|a_{[u]}\right| \\
= & (1+o(1)) N\left|a_{[u]}\right|-2 B(1+o(1))\left|a_{[u]}\right|,
\end{aligned}
$$

and similarly

$$
V_{u}^{u+N}(\alpha)<(1+o(1)) N\left|a_{[u]}\right|+2 B(1+o(1))\left|a_{[u]}\right| .
$$

Hence, one has only to choose $N=2 B+2$ and $C=4 B+3$ to derive the desired result.

Somewhat less immediate is the proof of the following theorem which is related to Theorem 4.2 . 
THEOREM 6.2. Under the conditions of Theorem 4.2 suppose that, furthermore, there exists a singularity $i \tau_{0}$ with which is associated a Taylor-D series $\phi_{\tau_{0}}(s)=\sum a_{n}^{\tau_{0}} e^{-n s}$ converging for $\operatorname{Re}\{s\}>0$ which not only satisfies (4.21) (with $h_{\tau_{0}}=1$ ) and is such that $f(s)-\phi_{\tau_{0}}(s)$ is regular at i $\tau_{0}$ but also

$$
\begin{aligned}
& \left|a_{n}^{\tau_{0}}\right| \geqq \delta q_{n} \text { for some } \delta>0 \text { and } n \text { sufficiently large, and } \\
& \lim _{n=\infty}\left(a_{n+1}^{\tau_{0}} / a_{n}^{\tau_{0}}\right)=e^{i \tau_{0}} .
\end{aligned}
$$

Then there exist two positive numbers $N$ and $C$ such that (6.1) holds.

Proof. The upper estimate in (6.1) was already established by Theorem 4.2. We need to show only that a corresponding lower bound exists. We shall again prove this by way of contradiction. Assuming that our contention (6.1) with regard to the lower bound (for some constant $C$ ) is false we conclude that there must exist a sequence of integers $\left\{n_{k}\right\}$ and a sequence of numbers $\left\{N_{k}\right\}, n_{k} \uparrow \infty$ and $N_{k} \uparrow \infty$, such that

$$
\int_{n_{k}}^{n_{k}+N_{k}}|d \alpha(u)|=o\left(a_{n_{k}}\right) \quad \text { as } k \rightarrow \infty .
$$

Let $h(s)=f(s)-\phi_{\tau_{0}}(s)$ and put

$$
h_{n_{k}}(s)=\frac{f(s)-\phi_{\tau_{0}}(s)-\left(\int_{0}^{n_{k}} e^{-s u} d \alpha(u)-\sum_{0}^{n_{k}-1} a_{\nu}^{\tau_{0}} e^{-\nu s}\right)}{a_{n_{k}} e^{-n_{k s}}}
$$

$$
\begin{aligned}
& =\frac{f(s)-\int_{0}^{n_{k}} e^{-s u} d \alpha(u)}{a_{n_{k}} e^{-n_{k s}}}-\frac{\phi_{\tau_{0}}(s)-\sum_{0}^{\tau_{k}-1} a_{\nu}^{\tau_{0}} e^{-\nu s}}{a_{n_{k}} e^{-n_{k} s}} \\
& =f_{n_{k}}(s)-\phi_{n_{k}}^{\tau_{0}}(s) .
\end{aligned}
$$

Using Theorem 4.2 (which gives $V_{u}^{u+1}(\alpha)=O\left(a_{[u]}\right)$ ) and applying Theorem 2.2 , one finds that the sequence $\left\{h_{n_{k}}(s)\right\}$ is uniformly bounded in any compact region where $h(s)$ is analytic. (This will be in particular true for a certain neighborhood of $i \tau_{0}$.) Let $\phi(s)$ be a limit function of the above sequence. One may write then for $\operatorname{Re}\{s\}>0$ that

$$
\begin{aligned}
\phi(s)= & \lim _{\boldsymbol{k}^{\prime}=\infty} f_{n_{k^{\prime}}}(s)-\lim _{\boldsymbol{k}^{\prime}=\infty} \phi_{n_{k^{\prime}}}^{\tau_{0}}(s)=\lim _{\boldsymbol{k}^{\prime}=\infty} \frac{1}{a_{n_{k^{\prime}}}} \int_{0}^{\infty} e^{-s u} d \alpha\left(n_{k^{\prime}}+u\right) \\
& -\lim _{\boldsymbol{k}^{\prime}=\infty} \sum_{\nu=0}^{\infty} \frac{a_{n_{k^{\prime}+\nu}+\nu}^{\tau_{0}}}{a_{n_{k^{\prime}} \tau^{\prime} l}^{\tau^{\prime}}} e^{-\nu s}
\end{aligned}
$$


( $\left\{k^{\prime}\right\}$ being a certain sequence of integers). But it is readily seen from (6.3) that $\lim f_{n_{k^{\prime}}}(s)=0$. Also, from (6.2) one sees that the last limit is $1 /\left(1-e^{i \tau_{0}-s}\right)$. That is, $\phi(s)=-1 /\left(1-e^{i \tau_{0}-s}\right)$. This contradicts our previous assertion that $\phi(s)$ is regular at $i \tau_{0}$ and proves the theorem.

Finally we shall state a third result which concerns Dirichlet series. We shall omit the proof of this theorem since it follows closely the proofs of the theorems given above.

THEOREM 6.3. Let $f(s)=\sum a_{n} e^{-\lambda_{n} s}$ satisfy the conditions of Theorem 4.3. Suppose, furthermore, that there exists on the imaginary axis a singular point $i \tau_{0}$ of $f(s)$ with which is associated a Taylor-D series $\phi_{\tau_{0}}(s)=\sum a_{n}^{\tau_{0}} e^{-n s}$ converging for $\operatorname{Re}\{s\}>0$ and such that:

(i) $f(s)-\phi_{\tau_{0}}(s)$ is regular at $s_{0}=i \tau_{0}$.

(ii) $A^{-1} q_{n} \leqq\left|a_{n}^{\tau_{0}}\right| \leqq A q_{n}$ for $n$ sufficiently large and some constant $A,\left(\left\{q_{n}\right\}\right.$ being the regular sequence of Theorem (4.3)).

(iii) $\lim \left(a_{n+1}^{\tau_{0}} / a_{n}^{\tau_{0}}\right)=e^{i \tau_{0}}$.

Then there exist positive numbers $N$ and $C$ such that

$$
C^{-1} q_{\left[\lambda_{n}\right]} \leqq \max _{\left|\lambda_{k}-\lambda_{n}\right| \leqq N}\left|a_{k}\right| \leqq C q_{\left[\lambda_{n}\right]},
$$

for $n$ sufficiently large.

7. More refined Tauberians. We shall now show that if a certain additional condition is satisfied, then the two-sided inequality of the previous section can be replaced by an asymptotic relation. This condition will simply be that one singular point (say $s=0$ ) is the dominant singularity on the imaginary axis in a certain sense. Although we shall limit ourselves here to the case of one dominant singularity, we want to point out that other asymptotic relations can be established in case there is more than one dominant singularity. In the general case, however, the asymptotic relation is less simple and depends on the set of dominant singularities on the imaginary axis.

THEOREM 7.1. Let $f(s)$ be represented in the half-plane $\operatorname{Re}\{s\}>0$ by (1.4) where $\alpha(u)$ belongs to the class $\Delta$. Suppose that $s=0$ is the dominant singularity of $f(s)$ on the imaginary axis in the following sense: there exists a Taylor-D series $\phi_{0}(s)$ converging for $\operatorname{Re}\{s\}>0$ and such that

(i) $f(s)-\phi_{0}(s)$ is regular at the origin.

(ii) $\phi_{0}(s)$ admits a decomposition $\phi_{0}(s)=\phi(s)+\phi^{*}(s)=\sum a_{n} e^{-n s}+\sum a_{n}^{*} e^{-n s}$ for $\operatorname{Re}\{s\}>0$, such that $a_{n}^{*}=o\left(a_{n}\right)$ and $\delta q_{n} \leqq\left|a_{n}\right| \leqq q_{n}$ where $\left\{q_{n}\right\}$ is a sequence satisfying (2.18) and $\delta>0$.

(iii) The only singularities of $\phi(s)$ on the imaginary axis are the points $s=2 k \pi i(k=0, \pm 1, \cdots)$.

(iv) If it is any singularity of $f(s)$ on the imaginary axis different from 
$s=2 k \pi i$, then there exists a Taylor-D series $\phi_{\tau}(s)=\sum a_{n}^{\tau} e^{-n s}$ converging for $\operatorname{Re}\{s\}>0$, such that

$$
f(s)-\phi_{\tau}(s) \text { is regular at ir and } a_{n}^{\tau}=o\left(a_{n}\right) \text { as } n \rightarrow \infty .
$$

Then

$$
\alpha(u+l)-\alpha(u) \sim l a_{[u]}
$$$$
\text { as } u \rightarrow \infty \text {, }
$$

uniformly for $0<l_{1} \leqq l \leqq l_{2}\left(l_{1}\right.$ and $l_{2}$ being constants).

Proof. Let $\phi(s)$ be an analytic function represented in the half-plane $\operatorname{Re}\{s\}>0$ by

$$
\Phi(s)=\int_{0}^{\infty} e^{-s u} \psi(u) d u
$$

and such that

(a) The only singularity of $\bar{\phi}(s)$ on the imaginary axis is the point $s=0$.

(b) $\phi(s)-\phi(s)$ is regular at the origin.

(c) $\psi(u) \sim a_{[u]}$ as $u \rightarrow \infty$.

The existence of such a function (with $\psi(u)$ which is even entire function of exponential type) was established in Lemma 5.2. We shall show that

$$
\alpha(u)=c+\int_{0}^{u} \psi(x) d x+\sum_{n<u} a_{n}^{*}+o\left(a_{[u]}\right)
$$

where $c$ is a certain constant. It is easily seen that the result (7.1) of the lemma will follow from (7.3) as a particular case. Let us put $h(s)=f(s)-(\phi(s)$ $+\phi^{*}(s)$ ). From (i) and (b) we note that $h(s)$ is regular at the origin. Set

$$
\begin{gathered}
h_{x}(s)=\frac{h(s)-\left[\int_{0}^{x} e^{-s u} d \alpha(u)-\int_{0}^{x} e^{-s u} \psi(u) d u-\sum_{n<x} a_{n}^{*} e^{-n s}\right]}{a_{[x]} e^{-x s}} \\
=\frac{f(s)-\int_{0}^{x} e^{-s u} d \alpha(u)}{a_{[x]} e^{-x s}}-\frac{\phi(s)-\int_{0}^{x} e^{-s u} \psi(u) d u}{a_{[x]} e^{-x s}} \\
-\frac{\phi^{*}(s)-\sum_{n<x} a_{n}^{*} e^{-n s}}{a_{[x]} e^{-x s}}=f_{x}(s)-\phi_{x}(s)-\phi_{x}^{*}(s) .
\end{gathered}
$$

Since, now, by Theorem 4.2, $V_{u}^{u+1}(\alpha)=O\left(a_{[u]}\right)$, and since $\psi(u) \sim a_{[u]}$ and $a_{n}^{*}=o\left(a_{n}\right)$, we can apply Theorem 2.2 to conclude that $\left\{h_{x}(s)\right\}$ is uniformly bounded in any compact region where $h(s)$ is analytic. We shall now establish that 


$$
\lim h_{x}(s)=0
$$

at any point of regularity of $h(s)$. The desired result (7.3) will easily follow from (7.5). For, using (7.4), we shall get for $s=0$ :

$$
h(0)-\left[\alpha(x)-\alpha(0)-\int_{0}^{x} \psi(u) d u-\sum_{n<x} a_{n}^{*}\right]=o\left(a_{[x]}\right),
$$

which is nothing else but (7.3) if we let $c=h(0)+\alpha(0)$. Now, in establishing (7.5) it is clearly enough to show that all limit functions of $\left\{h_{x}(s)\right\}$ are identically zero. This we now proceed to show. Applying Theorem 3.1 we first note that any limit function $\phi(s)$ of the family is analytic in the halfplanes $\operatorname{Re}\{s\}>0$ and $\operatorname{Re}\{s\}<0$, and in a certain neighborhood of the origin. From (7.4) we get that

$$
\phi(s)=\lim _{k=\infty} f_{x_{k}}(s)-\lim _{\boldsymbol{k}=\infty} \bar{\phi}_{x_{k}}(s)-\lim _{\boldsymbol{k}=\infty} \boldsymbol{\phi}_{x_{k}}^{*}(s),
$$

where $x_{k} \uparrow \infty$. Now, since $a_{n}^{*}=o\left(a_{n}\right)$ it is readily seen that the last limit is zero. Also, since $\psi(u) \sim a_{[u]}$ and $a_{n} / a_{n+1}$ by Lemma 5.1 , we find that in the half-plane $\operatorname{Re}\{s\}>0$ :

$$
\lim _{k=\infty} \Phi_{x_{k}}(s)=\lim _{k=\infty} \int_{0}^{\infty} e^{-s u}\left(\frac{\psi\left(x_{k}+u\right)}{a_{\left[x_{k}\right]}}\right) d u=\int_{0}^{\infty} e^{-s u} d u=\frac{1}{s} .
$$

Thus, in the half-plane $\operatorname{Re}\{s\}>0$

$$
\phi(s)=\lim _{k=\infty} f_{x_{k}}(s)-\frac{1}{s} .
$$

From (7.6) we find, using the definition of $f_{x}(s)$ in (7.4), that

$$
\phi(s)=\int_{0}^{\infty} e^{-s u} d \beta_{+}(u)-\frac{1}{s} \quad \text { for } \operatorname{Re}\{s\}>0
$$

where

$$
\beta_{+}(u)=\lim _{k^{\prime}=\infty}\left(\frac{\alpha\left(u+x_{k^{\prime}}\right)-\alpha\left(x_{k^{\prime}}\right)}{a_{\left[x_{k^{\prime}}\right]}}\right)
$$

( $\left\{k^{\prime}\right\}$ being a subsequence of the integers). Similarly, in the half-plane $\operatorname{Re}\{s\}<0$ one gets

$$
\phi(s)=-\lim _{k=\infty}\left[\frac{\int_{0}^{x_{k}} e^{-s u} d \alpha(u)}{a_{\left[x_{k}\right]} e^{-s x_{k}}}-\frac{\int_{0}^{x_{k}} e^{-s u} \psi(u) d u}{a_{\left[x_{k}\right]} e^{-s x_{k}}}-\frac{\sum_{\nu<x_{k}} a_{\nu}^{*} e^{-\nu s}}{a_{\left[x_{k}\right]} e^{-s x_{k}}}\right] .
$$

Using again hypothesis (ii) one sees that the last term in the brackets tends to 
zero. Also, using (c),

$$
\begin{aligned}
\lim _{k=\infty}\left(\frac{\int_{0}^{x_{k}} e^{-s u} \psi(u) d u}{a_{\left[x_{k}\right]} e^{-s x_{k}}}\right) & =\lim _{k=\infty} \int_{0}^{x_{k}} e^{s u}\left(\frac{\psi\left(x_{k}-u\right)}{a_{\left[x_{k}\right]}}\right) d u \\
& =\int_{0}^{\infty} e^{s u} d u=-\frac{1}{s} .
\end{aligned}
$$

As before, this leads to the representation

$$
\phi(s)=-\int_{0}^{\infty} e^{s u} d \beta_{-}(u)-\frac{1}{s} \quad \text { for } \operatorname{Re}\{s\}<0,
$$

where

$$
\beta_{-}(u)=\lim _{k^{\prime}=\infty}\left(\frac{\alpha\left(x_{k^{\prime}}\right)-\alpha\left(x_{k^{\prime}}-u\right)}{a_{\left[x_{k^{\prime}}\right]}}\right) .
$$

Now, from the expression (7.6) it is obvious that $\phi(s)$ is regular at all regular points of $f(s)$ on the imaginary axis. As we have noted before it is also regular at the origin. We claim, furthermore, that it is also regular at all points $i \tau \neq 0$ which are singularities of $f(s)$. Indeed, let $\phi_{\tau}(s)$ be the associated Taylor- $D$ series of hypothesis (iv). Put

$$
h_{\tau}(s)=f(s)-\phi_{\tau}(s)
$$

and

$$
\begin{aligned}
h_{x}^{\tau}(s) & =\frac{h_{\tau}(s)-\left[\int_{0}^{x} e^{-s u} d \alpha(u)-\sum_{n<x} a_{n}^{\tau} e^{-n s}\right]}{a_{[x]} e^{-x s}} \\
& =\frac{f(s)-\int_{0}^{x} e^{-s u} d \alpha(u)}{a_{[x]} e^{-x s}}-\frac{\phi_{\tau}(s)-\sum_{n<x} a_{n}^{\tau} e^{-n s}}{a_{[x]} e^{-x s}}=f_{x}(s)-\phi_{x}^{\tau}(s) .
\end{aligned}
$$

Using $a_{n}^{\tau}=o\left(a_{n}\right)$ one finds that $\lim \phi_{x}^{\tau}(s)=0$. Combining (7.6) and (7.9) one obtains

$$
\phi(s)+\frac{1}{s}=\lim _{k \rightarrow \infty} f_{x_{k}}(s)=\lim _{k=\infty} h_{x_{k}}^{\tau}(s) .
$$

Thus, $\phi(s)+1 / s$ (which is independent of $\tau$ ) is a limit function of the family $\left\{h_{x}^{\tau}(s)\right\}$ which is normal at $i \tau$. This establishes our assertion. Summing up we find that $\phi(s)$ is analytic in the whole plane. Moreover, like all limit functions $\phi(s)$ discussed in this paper it satisfies an inequality of the type (3.17). 
It will follow from the above properties that $\phi(s)$ must be a constant $C$. We shall show now that $C=0$. To this end let $\phi_{1}(s)=\phi(s)+1 / s$. Then

$$
\begin{aligned}
& \phi_{1}(s)=C+\frac{1}{s}=C+\int_{0}^{\infty} e^{-s u} d u=\int_{0}^{\infty} e^{-s u} d \gamma_{+}(u) \quad \text { for } \operatorname{Re}\{s\}>0, \\
& \phi_{1}(s)=C+\frac{1}{s}=C-\int_{0}^{\infty} e^{s u} d u=-\int_{0}^{\infty} e^{s u} d \gamma_{-}(u) \text { for } \operatorname{Re}\{s\}<0,
\end{aligned}
$$

where

$$
\begin{array}{llll}
\gamma_{+}(0)=0 & \text { and } & \gamma_{+}(u)=u+C & \text { for } u>0, \\
\gamma_{-}(0)=0 & \text { and } & \gamma_{-}(u)=u-C & \text { for } u>0 .
\end{array}
$$

Comparing (7.7) and (7.8) with (7.10) and $\left(7.10^{\prime}\right)$ it follows from the unicity theorem for Laplace transforms (since $\beta_{ \pm}(0)=0$ ) that at all points of continuity $(u>0)$ of $\beta_{+}(u)$ and $\beta_{-}(u)$, respectively, one has

$$
\beta_{+}(u)=u+C \text { and } \beta_{-}(u)=u-C .
$$

Now, if $d$ is the constant associated with $\alpha(u)$ by Definition 3.1, it follows (using $\left(7.7^{\prime}\right),\left(7.8^{\prime}\right)$ and the fact that $\alpha(u)$ belongs to the class $\Delta$ ) that all the numbers $\beta_{+}(u)$ and $\beta_{-}(u)$ for $0 \leqq u \leqq d / 2$ lie in an angle of the complex plane having its vertex at the origin and whose opening is less than $\pi$. Letting $u$ tend to zero through points of continuity of $\beta_{+}(u)$ and $\beta_{-}(u)$, respectively, one sees that (7.11) will be compatible with our last statement only if $C=0$. This establishes (7.5) and with it the theorem.

We shall now show that the generalization of Ikehara's theorem mentioned in the Introduction (Theorem 1.2) is a particular case of Theorem 7.1. As a matter of fact we shall establish a somewhat more general theorem.

THEOREM 7.2. Let $f(s)$ be represented in the half-plane $\operatorname{Re}\{s\}>0$ by (1.4) where $\alpha(u)$ belongs to the class $\Delta$. Suppose that $f(s)$ is bounded in any strip $\sigma>0,0<\epsilon \leqq t \leqq T$ and $\sigma>0,-T \leqq t \leqq-\epsilon$. Suppose, furthermore, that there exists a Taylor-D series $\phi(s)=\sum a_{n} e^{-n s}$ converging for $\operatorname{Re}\{s\}>0$ and such that:

(i) $f(s)-\phi(s)$ is bounded in a certain half strip $\sigma>0,|t| \leqq T_{0}<\pi$.

(ii) The only singularities of $\phi(s)$ on the imaginary axis are the points $2 k \pi i(k=0, \pm 1, \cdots)$.

(iii) $\delta q_{n} \leqq\left|a_{n}\right| \leqq q_{n}$ where $\left\{q_{n}\right\}$ satisfies (2.18).

(iv) $\lim \inf \left|a_{n}\right|>0$.

Then

$$
\alpha(u+l)-\alpha(u) \sim l a_{[u]},
$$

uniformly for $0<l_{1} \leqq l \leqq l_{2}\left(l_{1}\right.$ and $l_{2}$ being arbitrary positive constants).

Proof. Theorem 7.2 will follow from Theorem 7.1 once we show that the functions $\phi_{0}(s)$ and $\phi_{r}(s)$ exist and satisfy all the necessary conditions. To 
this end put $h(s)=f(s)-\phi(s)$. From the boundedness of $h(s)$ in the halfstrip $\sigma>0,|t| \leqq T_{0}$ it follows that $\lim h(\sigma+i t)(\sigma \rightarrow+0)$ exists almost everywhere for $|t| \leqq T_{0}$. We shall write $h(i t)$ for this limit which is a bounded function in the interval. Let $C_{0}$ be the half-circle $|\zeta|=|\xi+i \eta|=T_{0}, \xi \geqq 0$, directed from $-i T_{0}$ to $i T_{0}$. Then, if $s$ is in the half-disc bounded by $C_{0}$ and the imaginary axis, we may write (using the residue theorem), since $0<T_{0}<\pi$,

$$
\begin{aligned}
h(s) & =-\frac{1}{2 \pi i} \int_{C_{0}} \frac{h(\zeta)}{1-e^{\zeta-s}} d \zeta+\frac{1}{2 \pi} \int_{-T_{0}}^{T_{0}} \frac{h(i \eta)}{1-e^{i \eta-s}} d \eta \\
& =h_{1}(s)+\phi^{*}(s) .
\end{aligned}
$$

Now it is readily seen that $h_{1}(s)$ which is defined by the first integral (7.13) is regular at the origin. Thus if we choose $\phi_{0}(s)=\phi(s)+\phi^{*}(s)$, then $f(s)-\phi_{0}(s)$ $=h_{1}(s)$ will be regular at the origin. Also, $\phi^{*}(s)$ admits in the half-plane $\operatorname{Re}\{s\}>0$ a Taylor $-D$ expansion:

$$
\phi^{*}(s)=\sum_{n=0}^{\infty} a_{n}^{*} e^{-n s},
$$

where

$$
a_{n}^{*}=\frac{1}{2 \pi} \int_{-T_{0}}^{T_{0}} h(i \eta) e^{i n \eta} d \eta .
$$

That is, $a_{n}^{*}$ is a Fourier coefficient of the bounded function $h(i t)$. Thus, $a_{n}^{*} \rightarrow 0$. On the other hand, by our assumption, $a_{n}$ is bounded away from zero. Consequently we have the desired property $a_{n}^{*}=o\left(a_{n}\right)$. This shows that $\phi_{0}(s)$ has all the required properties in Theorem 7.1. Similarly, if $i \tau \neq 0$ is a singularity of $f(s)$ and if $0<\epsilon<\pi$ is sufficiently small so that $[-\epsilon+\tau, \epsilon+\tau]$ excludes the origin, then $f(i t)$ (which is defined almost everywhere) is bounded in $[-\epsilon+\tau, \epsilon+\tau]$. Putting

$$
\phi_{\tau}(s)=\frac{1}{2 \pi} \int_{\tau-\epsilon}^{\tau+\epsilon} \frac{f(i \eta)}{1-e^{i \eta-s}} d \eta=\sum a_{n}^{\tau} e^{-n s} \text { for } \operatorname{Re}\{s\}>0,
$$

one shows easily as before that this function possesses all the properties required by Theorem 7.1. We have now only to apply this theorem to obtain the desired result (7.12).

As we have previously noted, Ikehara's theorem is a very special case of the last theorem. It is the case where $\phi(s)=A /\left(1-e^{-s}\right)=A+A e^{-s}+\cdots$, and where, furthermore, $\alpha(u)$ is monotonic.

In conclusion we want to insert an application of the more general result (7.3) arrived at in the process of proving Theorem 7.1. The result which we shall derive is a known one (e.g. [4, p. 58]; however, we obtain an estimate of the remainder term which is not discussed in [4]) and its interest lies mainly 
in the fact that it gives an indication of the type of results which one may establish using complex variable Tauberians only. We shall apply (7.3) to the logarithm of the Riemann zeta function. Let us first note that if in Theorem 7.1 the origin is an isolated singularity on the imaginary axis, then the function $\phi^{*}(s)$ can be dropped so that (7.3) is replaced by the simpler expression:

$$
\alpha(u)=c+\int_{0}^{u} \psi(u) d u+o\left(a_{[u]}\right),
$$

where the constant $c$ was shown to be equal to $\alpha(0)+h(0)$. Now, in our case we may write after a translation of variable:

$$
f(s)=\log \zeta(s+1)=\int_{0}^{\infty} e^{-s u} d \alpha(u) \quad \text { for } \operatorname{Re}\{s\}>0
$$

where

$$
\alpha(u)=\sum_{n<e^{u}} \frac{\Lambda_{1}(n)}{n} \quad\left(\Lambda_{1}(n)=1 / m \text { for } n=p^{m},=0 \text { otherwise }\right) .
$$

The function $\log \zeta(s+1)$ is regular in the half-plane $\operatorname{Re}\{s\} \geqq 0$ except for a logarithmic singularity at the origin, such that $\log \zeta(s+1)-\log (1 / s)$ is regular at the origin. Thus we see that all the conditions of Theorem 7.1 will be satisfied if we shall choose

$$
\phi(s)=\log \left(\frac{1}{1-e^{-s}}\right)=e^{-s}+\frac{e^{-2 s}}{2}+\cdots \quad \text { and } \quad \phi(s)=\int_{1}^{\infty} e^{-s u} \frac{d u}{u} .
$$

Applying (7.15) we shall find that

$$
\sum_{n<e^{u}} \frac{\Lambda_{1}(n)}{n}=\int_{1}^{u} \frac{d u}{u}+c+o\left(\frac{1}{u}\right)
$$

or

$$
\sum_{n<u} \frac{\Lambda_{1}(n)}{n}=\log \log u+c+o\left(\frac{1}{\log u}\right)
$$

Noting that

$$
\sum_{n<u} \frac{\Lambda_{1}(n)}{n}=\sum_{p<u} \frac{1}{p}+\sum_{m \geqq 2} \sum_{p^{m}<u} \frac{1}{m p^{m}}=\sum_{p<u} \frac{1}{p}+\sum_{m=2}^{\infty} \sum_{p} \frac{1}{m p^{m}}+O\left(\frac{1}{u}\right),
$$

we obtain

$$
\sum_{p<u} \frac{1}{p}=\log \log u+c_{0}+o\left(\frac{1}{\log u}\right) .
$$


We point out that in deriving (7.16) no growth properties of $\zeta(s)$ were used (compare $[4$, loc. cit. $]$ ).

\section{BIBLIOGRAPHY}

1. S. Agmon, Sur les séries de Dirichlet, Ann. Ecole Norm. vol. 66 (1949) pp. 263-310.

2. - Functions of exponential type in an angle and singularities of Taylor series, Trans. Amer. Math. Soc. vol. 70 (1951) pp. 492-508.

3. S. Ikehara, An extension of Landau's theorem in the analytic theory of numbers, Journal of Mathematics and Physics, Massachusetts Institute of Technology, vol. 10, pp. 1-12.

4. E. C. Titchmarsh, The theory of the Riemann zeta function, Oxford, 1951.

The Rice Institute,

Houston, Tex.

The Hebrew University,

JERUSALEM, ISRaEL. 